\title{
THE INTERFACE CONTROL DOMAIN DECOMPOSITION (ICDD) METHOD FOR ELLIPTIC PROBLEMS*
}

\author{
MARCO DISCACCIATI ${ }^{\dagger}$, PAOLA GERVASIO ${ }^{\ddagger}$, AND ALFIO QUARTERONI ${ }^{\S}$
}

\begin{abstract}
Interface controls are unknown functions used as Dirichlet or Robin boundary data on the interfaces of an overlapping decomposition designed for solving second order elliptic boundary value problems. The controls are computed through an optimal control problem with either distributed or interface observation. Numerical results show that, when interface observation is considered, the resulting interface control domain decomposition method is robust with respect to coefficients variations; it can exploit nonconforming meshes and provides optimal convergence with respect to the discretization parameters; finally it can be easily used to face heterogeneous advectionadvection-diffusion couplings.
\end{abstract}

Key words. domain decomposition, optimal control, elliptic boundary value problems, nonconforming discretizations, heterogeneous coupling, ICDD

AMS subject classifications. 65N55, 49J20, 49K20, 65N30, 65N35

DOI. $10.1137 / 120890764$

1. Introduction. In this paper we propose an overlapping domain decomposition method with interface conditions that are suitable to face both homogenenous and heterogeneous couplings, i.e., with either the same or different operators in the subdomains.

We start by considering a family of overlapping domain decomposition methods, originally proposed in [13] and [16], named least square conjugate gradient (LSCG) and virtual control (VC) methods, respectively, designed for second order self-adjoint elliptic problems. These methods reformulate the overlapping multidomain problem as an optimal control problem whose controls are the unknown traces (or fluxes) of the state solutions at the subdomain boundaries. (For this reason we speak about interface controls.) The constraints of the minimization problem are the state equations, the observation is distributed on the overlap, and the controls are found by either minimizing the $L^{2}$ or $H^{1}$ norm of the jump between state solutions associated with the subdomains that share the same overlap. When the optimal control problem is solved through the optimality system, by following the classical theory of Lions ([15]), we need to solve both the primal and the dual state problems, as well as to compute the jump between the two solutions on the whole overlap. When non-self-adjoint problems are considered, the matrices associated with the discretization of both primal and dual problems have to be built and stored; moreover the same computational grid on the overlap has to be used, in order to avoid heavy interpolation processes from one grid to another. As we will show in [6], the convergence rate of these methods

\footnotetext{
${ }^{\dagger}$ Laboratori de Càlcul Numèric (LaCàN), Escola Tècnica Superior d'Enginyers de Camins, Canals i Ports de Barcelona (ETSECCPB), Universitat Politècnica de Catalunya (UPC BarcelonaTech), E08034 Barcelona, Spain (marco.discacciati@upc.edu). This author acknowledges the support of the Marie Curie Career Integration grant 2011-294229 within the 7th European Community Framework Programme.

¥DICATAM, Università di Brescia, I-25123 Brescia, Italy (gervasio@ing.unibs.it).

$\S$ MOX, Politecnico di Milano, I-20133 Milano, Italy, and MATHICSE, Chair of Modeling and Scientific Computing, Ecole Polytechnique Fédérale de Lausanne, CH-1015 Lausanne, Switzerland (alfio.quarteroni@epfl.ch).
} 
depends on the discretization parameters (grid size and local polynomial degree) and the overlap thickness.

In the present paper we propose a new version of the above-mentioned methods in which the observation is of interface type (and not distributed on the overlap) and it is restricted to the internal boundaries of the overlapping subdomains (that we can call for sake of simplicity the interfaces).

In this paper we propose to rename the LSCG and VC methods as well as the new one, interface control domain decomposition (ICDD) method, the former two with distributed observation, the last one with interface observation.

ICDD with interface observation minimizes a suitable norm of the jump between the solutions of different subdomains at the interfaces. The associated Euler-Lagrange equation cannot be written in terms of the dual state solutions and the optimality system cannot be inferred in a classical way. Nevertheless, we prove that solving the Euler-Lagrange equation is equivalent to solve a pseudooptimality system, in which the dual differential problems are replaced by primal problems depending on the jump of the state solutions only at the interfaces, and the Euler-Lagrange equation can be replaced by a linear combination of the traces of the state solutions.

The resulting domain decomposition method is robust and efficient with respect to the variations of the coefficients of the problem, as our numerical results show. It is also easily adaptable to solve heterogeneous problems since it requires neither an in-depth (specific) knowledge of the differential subproblems nor specific interface conditions as happens for domain decomposition methods with sharp interfaces (see, e.g., $[2,4,8])$. In particular, in this paper the ICDD method with interface observation is successfully applied to the coupling between advection and advection-diffusion (A-AD) problems. Our numerical results show that the heterogeneous solution converges to the global elliptic one when the viscosity vanishes and that the computational cost for solving the heterogeneous problem is lower than that needed to solve the homogeneous one.

Finally, it is worth noticing that the ICDD method with interface observation works well as a nonconforming method, also for convection dominated problems. As a matter of fact, the discretizations inside different subdomains can be totally unrelated and the evaluation of the jump between the state solutions across the interfaces can be performed by an interpolation step with a computational cost that does not affect the global efficiency of the method. In particular, no mortar approach is needed to guarantee the optimal convergence with respect to the discretization parameters, as the numerical results of the last section show.

In this paper we limit ourselves to the present and study the well-posedness of ICDD methods, while we defer to forthcoming papers other relevant aspects of the topic. In particular, in [6] we analyze the efficiency of ICDD methods with respect to the discretization parameters, such as the number of subdomains, the thickness of the overlap, the mesh size (in the case of finite elements), or the polynomial degree (in the case of spectral elements). Moreover, in [5], we compare ICDD methods with the more classical additive Schwarz method with coarse grid when elliptic self-adoint problems are taken into account.

In [7] we apply the ICDD method with interface observation to the heterogeneous coupling between Stokes and Darcy equations for the simulation of fluids in porous media.

An outline of this paper is as follows.

In section 2 we give two multidomain formulations of the reference elliptic differential problem and we prove their equivalence. These formulations stand at the base 
of ICDD methods. In section 3 we present ICDD methods and in section 4 we prove that the minimization problems (from which ICDD arises) are well-posed. Then we formulate the optimality systems (pseudooptimality system when interface observation is considered) which are the kernels for practical implementation of the methods themselves. In section 5 we extend the ICDD method with interface observation to the A-AD coupling and we mention the heterogeneous Stokes-Darcy coupling that will be analyzed in depth in [7]. Finally, in section 6 we report numerical results on two-dimensional (2D) test cases approximated by both finite elements and spectral elements.

2. Problem setting. Let $\Omega \subset \mathbb{R}^{d}(d=1,2,3)$ be an open bounded domain. We split $\Omega$ into two overlapping subdomains $\Omega_{1}$ and $\Omega_{2}$ such that $\Omega=\Omega_{1} \cup \Omega_{2}$ and we denote $\Omega_{12}=\Omega_{1} \cap \Omega_{2}$ and $\Gamma_{i}=\partial \Omega_{i} \backslash \partial \Omega$. Moreover, let $\partial \Omega=\Gamma_{D} \cup \Gamma_{N}$ with $\Gamma_{D} \cap \Gamma_{N}=\emptyset$ as shown in Figure 2.1, and denote $\Gamma_{D}^{i}=\Gamma_{D} \cap \partial \Omega_{i}$ and $\Gamma_{N}^{i}=\Gamma_{N} \cap \partial \Omega_{i}$.

Let $L$ be the linear elliptic operator

$$
L u=\operatorname{div}(-K \nabla u+\mathbf{b} u)+b_{0} u,
$$

where $K=K(x)$ is a symmetric positive definite tensor $K=\left(K_{i j}\right)_{i, j=1, \ldots, d}, K_{i j} \in$ $L^{\infty}(\Omega), K_{i j}=K_{j i}$, that satisfies the ellipticity constraint

$$
\sum_{i, j=1}^{d} K_{i j} \xi_{i} \xi_{j} \geq K|\boldsymbol{\xi}|^{2} \quad \forall \boldsymbol{\xi}=\left(\xi_{1}, \ldots, \xi_{d}\right) \in \mathbb{R}^{d}
$$

for a suitable $K>0$.

We consider the following boundary value problem.

Global problem $\mathcal{P}$.

$$
\begin{aligned}
L u & =f & & \text { in } \Omega, \\
u & =\phi_{D} & & \text { on } \Gamma_{D}, \\
\partial_{n_{L}} u & =\phi_{N} & & \text { on } \Gamma_{N},
\end{aligned}
$$

where $f \in L^{2}(\Omega), \phi_{D} \in H^{1 / 2}\left(\Gamma_{D}\right)$, and $\phi_{N} \in H^{-1 / 2}\left(\Gamma_{N}\right)$ are assigned functions satisfying suitable compatibility conditions on $\Gamma_{N} \cap \Gamma_{D}$ (see [14]), and $\partial_{n_{L}} u$ denotes the conormal derivative of $u$ :

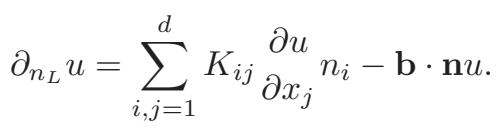

$n_{i}$ are the components of $\mathbf{n}$, the latter being the unit normal vector external to $\partial \Omega$.
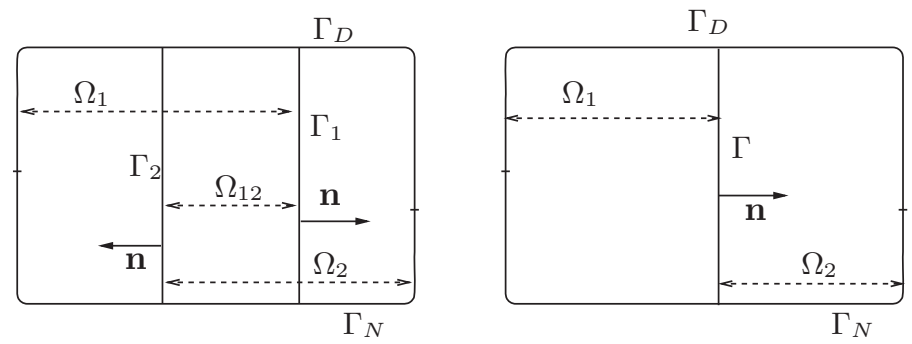

FIG. 2.1. Partition of $\Omega$ into two subdomains. On the left is the overlapping case, on the right the nonoverlapping one. 
A few technical assumptions on $\mathbf{b}$ and $b_{0}$ are made in order to guarantee the well-posedness of (2.2), besides requiring that $\mathbf{b} \in\left[W^{1, \infty}(\Omega)\right]^{d}$ and $b_{0} \in L^{\infty}(\Omega)$, with $b_{0}(\mathbf{x}) \geq 0$ in $\Omega$ :

1. if $\Gamma_{N}=\emptyset, \exists \sigma_{0} \geq 0$ such that

$$
b_{0}(\mathbf{x})+\frac{1}{2} \operatorname{div} \mathbf{b}(\mathbf{x}) \geq \sigma_{0} \quad \forall \mathbf{x} \in \Omega ;
$$

2. if $\Gamma_{D}=\emptyset, \exists \sigma_{0}>0$ such that (2.3) is satisfied and moreover

$$
\exists \varepsilon_{0} \geq 0 \text { such that }\|\mathbf{b}\|_{L^{\infty}\left(\Gamma_{N}\right)} \leq \frac{2\left[\min \left\{K, \sigma_{0}\right\}-\varepsilon_{0}\right]}{C^{*}},
$$

where $C^{*}$ is the constant of the trace inequality

$$
\|v\|_{L^{2}(\partial \Omega)}^{2} \leq C^{*}\|v\|_{H^{1}(\Omega)}^{2} \forall v \in H^{1}(\Omega) ;
$$

3. if both $\Gamma_{D} \neq \emptyset$ and $\Gamma_{N} \neq \emptyset, \exists \sigma_{0} \geq 0$ such that (2.3) is satisfied and $\exists \varepsilon_{0} \geq 0$ such that

(a) if $\sigma_{0}=0$, b satisfies

$$
\|\mathbf{b}\|_{L^{\infty}\left(\Gamma_{N}\right)} \leq \begin{gathered}
2 K C_{\Omega}-\varepsilon_{0} \\
C^{*}
\end{gathered}
$$

where $C_{\Omega}$ is the constant of the Poincaré inequality

$$
\|v\|_{L^{2}(\Omega)} \leq C_{\Omega}\|\nabla v\|_{L^{2}(\Omega)},
$$

(b) if $\sigma_{0}>0$, b satisfies (2.4),

then problem (2.2) admits a unique weak solution (see problem (2.13) below).

There are several ways to reformulate (2.2) in an equivalent multidomain manner. One possibility is on nonoverlapping subdomains $\Omega_{1}, \Omega_{2} \subset \Omega$, with $\Omega_{1} \cap \Omega_{2}=\emptyset$, $\Omega_{1} \cup \Omega_{2}=\Omega, \Gamma=\partial \Omega_{1} \cap \partial \Omega_{2}$, and it reads (see, e.g., [19])

$$
\begin{aligned}
& L u_{1}=f \quad \text { in } \Omega_{1}, \\
& L u_{2}=f \quad \text { in } \Omega_{2}, \\
& u_{1}=u_{2}, \quad \partial_{n_{L}} u_{1}=\partial_{n_{L}} u_{2} \quad \text { on } \Gamma
\end{aligned}
$$

with boundary conditions

$$
\begin{aligned}
u_{i}=\phi_{D \mid \Gamma_{D}^{i}} & \text { on } \partial \Omega_{i} \cap \Gamma_{D}, i=1,2, \\
\partial_{n_{L}} u_{i}=\phi_{N \mid \Gamma_{N}^{i}} & \text { on } \partial \Omega_{i} \cap \Gamma_{N}, i=1,2 .
\end{aligned}
$$

In the case of overlapping subdomains, we consider two different formulations. Although they are mathematically equivalent, they will give rise to two different ICDD methods that we compare in the forthcoming paper [6].

Problem $\mathcal{P}_{\Omega_{12}}$.

$$
\begin{aligned}
& L u_{1}=f \quad \text { in } \Omega_{1} \text {, } \\
& L u_{2}=f \quad \text { in } \Omega_{2} \text {, } \\
& u_{1}=u_{2} \quad \text { in } \Omega_{12}
\end{aligned}
$$

with boundary conditions (2.7); 


\section{Problem $\mathcal{P}_{\Gamma_{1} \cup \Gamma_{2}}$.}

$$
\begin{aligned}
L u_{1} & =f & & \text { in } \Omega_{1}, \\
L u_{2} & =f & & \text { in } \Omega_{2}, \\
\Psi\left(u_{1}\right) & =\Psi\left(u_{2}\right) & & \text { on } \Gamma_{1} \cup \Gamma_{2}
\end{aligned}
$$

with boundary conditions (2.7).

We denote by $\Psi\left(u_{i}\right)$ either the trace of $u_{i}$ on $\Gamma_{1} \cup \Gamma_{2}$, or its conormal derivative $\partial_{n_{L}} u_{i}$ on $\Gamma_{1} \cup \Gamma_{2}$, or else a linear combination between $u_{i}$ and $\partial_{n_{L}} u_{i}$. Thus, depending on the choice of $\Psi$, condition $(2.9)_{3}$ may become either

$$
u_{1}=u_{2} \quad \text { on } \Gamma_{1} \cup \Gamma_{2},
$$

(which stands at the base of the Schwarz method) or

$$
\partial_{n_{L}} u_{1}=\partial_{n_{L}} u_{2} \quad \text { on } \Gamma_{1} \cup \Gamma_{2},
$$

or else

$$
\beta u_{1}+\partial_{n_{L}} u_{1}=\beta u_{2}+\partial_{n_{L}} u_{2} \quad \text { on } \Gamma_{1} \cup \Gamma_{2},
$$

where $\beta \geq 0$ is a suitable parameter. The equality (2.11) on $\Gamma_{1}$ should be understood as follows. The normal vector $\mathbf{n}$ on $\Gamma_{1}$ is directed outward of $\Omega_{1}$ and the conormal derivative of $u_{2}$ is computed upon restricting $u_{2}$ to $\Omega_{2} \backslash \Omega_{12}$. On the other hand, on $\Gamma_{2}$ the normal vector $\mathbf{n}$ is directed outward of $\Omega_{2}$ and the conormal derivative of $u_{1}$ is taken upon restricting it to $\Omega_{1} \backslash \Omega_{12}$.

Since (2.11) is a special case of (2.12), in the following we will consider only the more general condition (2.12).

Let us denote by $R_{\phi_{D}}$ any lifting in $\Omega$ of the Dirichlet data $\phi_{D}$, that is $R_{\phi_{D}} \in$ $H^{1}(\Omega),\left.R_{\phi_{D}}\right|_{\Gamma_{D}}=\phi_{D}$. Analogously, $R_{i, \phi_{D}}$ denotes the lifting in $\Omega_{i}$ of $\left.\phi_{D}\right|_{\Gamma_{D}^{i}}$, for $i=1,2$.

We can associate problem (2.2) with the corresponding weak formulation: find $\left(u-R_{\phi_{D}}\right) \in V$ such that

$$
a(u, v)=F(v) \quad \forall v \in V,
$$

where $V=\left\{v \in H^{1}(\Omega): v=0\right.$ on $\left.\Gamma_{D}\right\}$, while the bilinear form $a(\cdot, \cdot)$ and the linear functional $F$ are defined as:

$$
\begin{aligned}
a(u, v) & =\int_{\Omega}(K \nabla u-\mathbf{b} u) \cdot \nabla v+\int_{\Omega} b_{0} u v \quad \forall u, v \in H^{1}(\Omega), \\
F(v) & =\int_{\Omega} f v+\int_{\Gamma_{N}} \phi_{N} v \quad \forall v \in H^{1}(\Omega) .
\end{aligned}
$$

To write the weak form of the local problems in (2.9), we introduce the Hilbert spaces

$$
V_{i}=\left\{v_{i} \in H^{1}\left(\Omega_{i}\right): v_{i}=0 \text { on } \Gamma_{D}^{i}\right\} \quad \text { and } \quad V_{i}^{D}=\left\{v_{i} \in V_{i}: v_{i}=0 \text { on } \Gamma_{i}\right\}
$$

endowed with the canonical norm of $H^{1}\left(\Omega_{i}\right)$, and we distinguish the case in which $(2.9)_{3}$ corresponds to (2.10) or (2.12). In the first case we have

find $\left(u_{i}-R_{i, \phi_{D}}\right) \in V_{i}$ such that

$$
a_{i}\left(u_{i}, v_{i}\right)=F_{i}\left(v_{i}\right) \quad \forall v_{i} \in V_{i}^{D}, \quad u_{i}=u_{j} \text { on } \Gamma_{i}, j \neq i, \quad i, j=1,2,
$$


where $a_{i}(\cdot, \cdot)$ is the restriction of the bilinear form $a(\cdot, \cdot)$ to $\Omega_{i}$, while

$$
F_{i}\left(v_{i}\right)=\int_{\Omega_{i}} f v_{i}+\int_{\Gamma_{N}^{i}} \phi_{N} v_{i} \quad \forall v_{i} \in V_{i}
$$

In the second case the weak form reads

find $\left(u_{i}-R_{i, \phi_{D}}\right) \in V_{i}$ such that

$a_{i}\left(u_{i}, v_{i}\right)+\int_{\Gamma_{i} \cup \Gamma_{N}^{i}} \beta u_{i} v_{i}-\int_{\Gamma_{i}}\left(\beta u_{j}+\partial_{n_{L}} u_{j}\right) v_{i}=F_{i}\left(v_{i}\right) \quad \forall v_{i} \in V_{i}, \quad i, j=1,2, j \neq i$.

The bilinear form $a(\cdot, \cdot)$ is continuous and also coercive on $V$. Indeed, the coercivity is guaranteed in the different cases by assumptions (2.3), (2.4), and (2.5).

Analogous results hold for the bilinear forms $a_{i}(\cdot, \cdot)$ on $V_{i}$.

We want now to prove that $(2.2),(2.8)$, and (2.9) are all equivalent problems. We introduce the affine manifolds

$$
V_{\phi_{D}}=\left\{v \in H^{1}(\Omega): v=\phi_{D} \text { on } \Gamma_{D}\right\}, \quad V_{i, \phi_{D}}=\left\{v_{i} \in H^{1}\left(\Omega_{i}\right): v_{i}=\phi_{D} \text { on } \Gamma_{D}^{i}\right\} .
$$

Proposition 2.1. The function $u \in V_{\phi_{D}}$ is the solution of (2.2) if and only if $u_{1}=\left.u\right|_{\Omega_{1}} \in V_{1, \phi_{D}}$ and $u_{2}=\left.u\right|_{\Omega_{2}} \in V_{2, \phi_{D}}$ are the solutions of (2.8). Moreover, $u_{1} \in V_{1, \phi_{D}}$ and $u_{2} \in V_{2, \phi_{D}}$ are the solutions of (2.8) if and only if they are solutions of $(2.9)$.

Proof. If $u \in V_{\phi_{D}}$ is the solution of (2.2), then the restrictions of $u$ to $\Omega_{1}$ and $\Omega_{2}$, $u_{\mid \Omega_{1}}$ and $u_{\mid \Omega_{2}}$, respectively, satisfy (2.8) by construction, so that we can set $u_{i}=u_{\mid \Omega_{i}}$.

Vice versa, if $u_{1}$ and $u_{2}$ are the solutions of (2.8), if we set

$$
u= \begin{cases}u_{1} & \text { in } \Omega_{1} \backslash \Omega_{12}, \\ u_{1}=u_{2} & \text { in } \Omega_{12}, \\ u_{2} & \text { in } \Omega_{2} \backslash \Omega_{12},\end{cases}
$$

it is straightforward to see that $u \in V_{\phi_{D}}$ and that it satisfies (2.2).

Assume that $u_{1}$ and $u_{2}$ are the solutions of problem (2.9). If we take $w=$ $u_{1 \mid \Omega_{12}}-u_{2 \mid \Omega_{12}}$ in $\Omega_{12}$, then by definition $w \in V_{12}$ with $V_{12}=\left\{v \in H^{1}\left(\Omega_{12}\right): v=\right.$ 0 on $\left.\partial \Omega_{12} \cap \Gamma_{D}\right\}$ and it satisfies the following problem (with homogeneous data):

$$
\begin{array}{rlrl}
L w=0 & & \text { in } \Omega_{12}, \\
\Psi(w) & =0 & & \text { on } \Gamma_{1} \cup \Gamma_{2}, \\
w & =0 & & \text { on } \Gamma_{D} \cap \partial \Omega_{12}, \\
\partial_{n_{L}} w & =0 & & \text { on } \Gamma_{N} \cap \partial \Omega_{12},
\end{array}
$$

whose unique solution is obviously $w=0$. As a consequence, $u_{1}=u_{2}$ in $\Omega_{12}$ and they satisfy problem (2.8).

Vice versa, if $u_{1}$ and $u_{2}$ satisfy (2.8), they also satisfy $(2.9)_{1},(2.9)_{2}$, and the boundary condition (2.10). Finally, since $u_{1} \in V_{1, \phi_{D}}, u_{2} \in V_{2, \phi_{D}}$, and $u_{1}=u_{2}$ in $\Omega_{12}$, their image on $\Gamma_{1} \cup \Gamma_{2}$ through the operator $\Psi$ are also equal.

Corollary 2.2. Problems (2.2), (2.8), and (2.9) are well-posed.

Proof. This follows from the well-posedness of (2.2) and the previous equivalence results. 
3. The ICDD method. In order to simplify our analysis, we consider problem (2.2) with homogeneous Dirichlet conditions, that is we put $\Gamma_{N}=\emptyset, \Gamma_{D}=\partial \Omega$, and $\phi_{D}=0$. As previously remarked, nonhomogeneous boundary data translate into a modified right-hand side thanks to the use of a suitable lifting operator. This problem modification does not affect the forthcoming analysis and discussion.

Then, we consider the overlapping decomposition of $\Omega$ introduced before.

The basic idea of the ICDD approach consists in introducing two controls which play the role of unknown Dirichlet (or Robin) data at the interfaces of the decomposition and in minimizing the difference between the solutions $u_{1}$ and $u_{2}$ of either problem (2.8) and (2.9) through a suitable cost functional defined in $\Omega_{12}$ or on $\Gamma_{1} \cup \Gamma_{2}$.

To this aim, for $i=1,2$, let us introduce the following vector spaces:

(i) admissible Dirichlet controls:

(ii) admissible Robin controls:

$$
\Lambda_{i}^{R}=\left(H_{00}^{1 / 2}\left(\Gamma_{i}\right)\right)^{\prime}
$$
spaces.

The spaces $\left(\Lambda_{i}^{D},\|\cdot\|_{H_{00}^{1 / 2}\left(\Gamma_{i}\right)}\right)$ and $\left(\Lambda_{i}^{R},\|\cdot\|_{\left(H_{00}^{1 / 2}\left(\Gamma_{i}\right)\right)^{\prime}}\right)$, for $i=1,2$, are Hilbert

In the case of Dirichlet controls, for $i=1,2$, we consider two unknown control functions $\lambda_{i} \in \Lambda_{i}^{D}$ and we introduce the state problems

$$
\begin{aligned}
L u_{i}=f & & \text { in } \Omega_{i}, \\
u_{i}=\lambda_{i} & & \text { on } \Gamma_{i}, \\
u_{i}=0 & & \text { on } \Gamma_{D}^{i} .
\end{aligned}
$$

In the case of Robin controls, for any $\beta \geq 0,(3.3)$ is replaced by

$$
\begin{aligned}
L u_{i} & =f & & \text { in } \Omega_{i}, \\
\beta u_{i}+\partial_{n_{L}} u_{i} & =\lambda_{i} & & \text { on } \Gamma_{i}, \\
u_{i} & =0 & & \text { on } \Gamma_{D}^{i},
\end{aligned}
$$

where now $\lambda_{i} \in \Lambda_{i}^{R}$ are the Robin controls.

The unknown interface controls are determined through the solution of a minimization problem involving a suitable cost functional depending on the difference, with respect to a suitably chosen norm, between $u_{1}$ and $u_{2}$ either on the overlapping region $\Omega_{12}$ (thus following $(2.8)_{3}$ ) or on the interface $\Gamma_{1} \cup \Gamma_{2}$ (referring to (2.9) $)_{3}$ ). For instance, taking $\lambda_{1}$ and $\lambda_{2}$ as Dirichlet interface controls, we denote by $u_{i}\left(\lambda_{i}\right)$ the solutions of (3.3) and we can proceed as follows.

Case 1. Minimization in the norm of $L^{2}\left(\Omega_{12}\right)$ :

$$
\inf _{\lambda_{1}, \lambda_{2}}\left[J_{0}\left(\lambda_{1}, \lambda_{2}\right)=\frac{1}{2}\left\|u_{1}\left(\lambda_{1}\right)-u_{2}\left(\lambda_{2}\right)\right\|_{L^{2}\left(\Omega_{12}\right)}^{2}\right] .
$$

Case 2. Minimization in the norm of $H^{1}\left(\Omega_{12}\right)$ :

$$
\inf _{\lambda_{1}, \lambda_{2}}\left[J_{1}\left(\lambda_{1}, \lambda_{2}\right)=\frac{1}{2}\left\|u_{1}\left(\lambda_{1}\right)-u_{2}\left(\lambda_{2}\right)\right\|_{H^{1}\left(\Omega_{12}\right)}^{2}\right] .
$$

Case 3. Minimization in the norm of $L^{2}\left(\Gamma_{1} \cup \Gamma_{2}\right)$ :

$$
\inf _{\lambda_{1}, \lambda_{2}}\left[J_{0, \Gamma}\left(\lambda_{1}, \lambda_{2}\right)={ }_{2}^{1}\left\|u_{1}\left(\lambda_{1}\right)-u_{2}\left(\lambda_{2}\right)\right\|_{L^{2}\left(\Gamma_{1} \cup \Gamma_{2}\right)}^{2}\right] .
$$


Remark 3.1. In the case $\Gamma_{N} \neq \emptyset$ and $\partial \Omega_{12} \cap \Gamma_{D} \neq \emptyset$, we can replace the functional $J_{1}$ in (3.6) by the equivalent one

$$
J_{|\cdot|_{1}}\left(\lambda_{1}, \lambda_{2}\right)={ }_{2}^{1}\left|u_{1}\left(\lambda_{1}\right)-u_{2}\left(\lambda_{2}\right)\right|_{H^{1}\left(\Omega_{12}\right)}^{2}
$$

The minimization problems (3.5), (3.6), and (3.7) with constraints (3.3) (or (3.4)) are in fact optimal control problems and they can be analyzed by using the classical theory of optimization (see, e.g., [15]). The controls are of boundary type (actually they are interface controls) while the observation is distributed on the overlap in both (3.5) and (3.6), while it is of boundary type in (3.7).

Problems (3.5) and (3.6) with constraints (3.3) were proposed in the papers by Glowinski, Dinh, and Periaux [13] and Lions and Pironneau [16], without, however, being analyzed. In [13] these methods were called least-squares conjugate-gradient methods, while in [16] they were named virtual control methods. The latter nomenclature has been used also by the authors of this paper in previous works (see $[12,4]$ ). In the next sections we will carry out the analysis of the minimization problems (3.5), (3.6), and (3.7) as well as the derivation of the associated optimality systems. Let us begin with the case of Dirichlet controls at the interfaces.

4. Analysis of the optimal control problems with Dirichlet interface controls. We define the Hilbert spaces $\mathbf{V}=V_{1} \times V_{2}, \mathbf{V}^{D}=V_{1}^{D} \times V_{2}^{D}$, and $\boldsymbol{\Lambda}^{D}=$ $\Lambda_{1}^{D} \times \Lambda_{2}^{D}$ endowed with the corresponding graph norms. Let $u_{i}^{\lambda_{i}, f}$ denote the solutions of problems (3.3) for $i=1,2$, and set $\mathbf{u}^{\boldsymbol{\lambda}, f}=\left(u_{1}^{\lambda_{1}, f}, u_{2}^{\lambda_{2}, f}\right)$ for any $\boldsymbol{\lambda}=\left(\lambda_{1}, \lambda_{2}\right) \in \boldsymbol{\Lambda}^{D}$.

Moreover, we set $\Lambda_{12}^{D}=L^{2}\left(\Gamma_{1} \cup \Gamma_{2}\right)$.

For the sake of notation unification, we can write (3.5)-(3.7) under the unified form

$$
\inf _{\boldsymbol{\lambda} \in \boldsymbol{\Lambda}^{D}}\left[J_{C, \mathcal{H}}(\boldsymbol{\lambda})={ }_{2}^{1}\left\|C \mathbf{u}^{\boldsymbol{\lambda}, f}\right\|_{\mathcal{H}}^{2}\right]
$$

where $\left(\mathcal{H},\|\cdot\|_{\mathcal{H}}\right)$ is a Hilbert space and $C: \mathbf{V} \rightarrow \mathcal{H}$ is a linear and continuous (observation) operator. The different cases are specified in Table 4.1 and the reason for the presence of the norms in the last column of the Table 4.1 will be clear after the proof of Lemma 4.1.

The function $\left.\left(u_{1}^{\lambda_{1}, f}-u_{2}^{\lambda_{2}, f}\right)\right|_{\Gamma_{1} \cup \Gamma_{2}}$ has to be interpreted in the sense of zeroth order trace in the space $H_{00}^{1 / 2}\left(\Gamma_{1} \cup \Gamma_{2}\right)$.

Because of problem linearities, $u_{i}^{\lambda_{i}, f}=u_{i}^{\lambda_{i}, 0}+u_{i}^{0, f}$; moreover, we denote by $u_{i}^{\lambda_{i}}=u_{i}^{\lambda_{i}, 0}$ the solution of (3.3) with $f=0$ and we set $\mathbf{u}^{\boldsymbol{\lambda}}=\left(u_{1}^{\lambda_{1}}, u_{2}^{\lambda_{2}}\right)$, so that the cost functionals can be written also as

$$
J_{C, \mathcal{H}}(\boldsymbol{\lambda})={ }_{2}^{1}\left\|C \mathbf{u}^{\boldsymbol{\lambda}}\right\|_{\mathcal{H}}^{2}+\left(C \mathbf{u}^{\boldsymbol{\lambda}}, C \mathbf{u}^{\mathbf{0}, f}\right)_{\mathcal{H}}+{ }_{2}^{1}\left\|C \mathbf{u}^{\mathbf{0}, f}\right\|_{\mathcal{H}}^{2}
$$

TABLE 4.1

Different items for problem (4.1) and their relative notations.

$\begin{array}{lllll} & J_{C, \mathcal{H}}(\boldsymbol{\lambda}) & \mathcal{H} & C \mathbf{u}^{\boldsymbol{\lambda}, f} & \|\cdot\|_{*} \\ \text { Case 1: } & J_{0}\left(\lambda_{1}, \lambda_{2}\right) & L^{2}\left(\Omega_{12}\right) & \left.\left(u_{1}^{\lambda_{1}, f}-u_{2}^{\lambda_{2}, f}\right)\right|_{\Omega_{12}} & \|\boldsymbol{\lambda}\|_{0}=\left\|C \mathbf{u}^{\boldsymbol{\lambda}}\right\|_{L^{2}\left(\Omega_{12}\right)} \\ \text { Case 2: } & J_{1}\left(\lambda_{1}, \lambda_{2}\right) & H^{1}\left(\Omega_{12}\right) & \left.\left(u_{1}^{\lambda_{1}, f}-u_{2}^{\lambda_{2}, f}\right)\right|_{\Omega_{12}} & \|\boldsymbol{\lambda}\|_{1}=\left\|C \mathbf{u}^{\boldsymbol{\lambda}}\right\|_{H^{1}\left(\Omega_{12}\right)} \\ \text { Case 3: } & J_{0, \Gamma}\left(\lambda_{1}, \lambda_{2}\right) & \Lambda_{12}^{D} & \left.\left(u_{1}^{\lambda_{1}, f}-u_{2}^{\lambda_{2}, f}\right)\right|_{\Gamma_{1} \cup \Gamma_{2}} & \|\boldsymbol{\lambda}\|_{0, \Gamma}=\left\|C \mathbf{u}^{\boldsymbol{\lambda}}\right\|_{L^{2}\left(\Gamma_{1} \cup \Gamma_{2}\right)}\end{array}$


Lemma 4.1. In all Cases $1-3,\left\|C \mathbf{u}^{\boldsymbol{\lambda}}\right\|_{\mathcal{H}}$ is a norm on the control space $\boldsymbol{\Lambda}^{D}$.

Proof. $\left\|C \mathbf{u}^{\boldsymbol{\lambda}}\right\|_{\mathcal{H}}$ is a seminorm on $\boldsymbol{\Lambda}^{D}$ in every case. Then we can limit ourselves to prove that if $\left\|C \mathbf{u}^{\boldsymbol{\lambda}}\right\|_{\mathcal{H}}=0$ then $\boldsymbol{\lambda}=\left(\lambda_{1}, \lambda_{2}\right)=\mathbf{0}$.

(i) We start by considering Case 1 , where $\mathcal{H}=L^{2}\left(\Omega_{12}\right)$ and $C \mathbf{u}^{\boldsymbol{\lambda}}=\left.\left(u_{1}^{\lambda_{1}}-u_{2}^{\lambda_{2}}\right)\right|_{\Omega_{12}}$. By using the same arguments adopted in the proof of Proposition 2.1, we can prove that $u_{i}^{\lambda_{i}}=0$ in $\Omega_{i}$ and then $\lambda_{i}=0$ on $\Gamma_{i}$ for $i=1,2$.

(ii) In Case $2, \mathcal{H}=H^{1}\left(\Omega_{12}\right)$ and $C \mathbf{u}^{\boldsymbol{\lambda}}=\left.\left(u_{1}^{\lambda_{1}}-u_{2}^{\lambda_{2}}\right)\right|_{\Omega_{12}}$ and the proof is similar.

(iii) In Case 3 , if $\left\|u_{1}^{\lambda_{1}}-u_{2}^{\lambda_{2}}\right\|_{L^{2}\left(\Gamma_{1} \cup \Gamma_{2}\right)}^{2}=0$ then $u_{1}^{\lambda_{1}}=u_{2}^{\lambda_{2}}$ a.e. on $\Gamma_{1} \cup \Gamma_{2}$; the $H^{1}$-regularity of both $u_{1}^{\lambda_{1}}$ and $u_{2}^{\lambda_{2}}$ implies $u_{i}^{\lambda_{i}} \in H_{00}^{1 / 2}\left(\Gamma_{i}\right)$ and then $\| u_{1}^{\lambda_{1}}-$ $u_{2}^{\lambda_{2}} \|_{H^{1 / 2}\left(\partial \Omega_{12}\right)}^{2}=0$. The thesis follows by the equivalence between $H^{1 / 2}(\partial \Omega)$ and $H^{1}(\Omega)$ norms and by (ii).

In view of the previous lemma, we can define three new norms in $\Lambda^{D}$ by setting $\|\boldsymbol{\lambda}\|_{*}=\left\|C \mathbf{u}^{\boldsymbol{\lambda}}\right\|_{\mathcal{H}}$ (those listed in the fourth column of Table 4.1).

It is not guaranteed that $\boldsymbol{\Lambda}^{D}$ is complete with respect to any of these norms. Nevertheless, it is possible to built the completions of $\left(\boldsymbol{\Lambda}^{D},\|\cdot\|_{*}\right)$ (see, e.g., [21]), with $\|\cdot\|_{*}$ chosen between those of Table 4.1 and to look for the solution of the minimization problem (4.3) in such a complete space. The abstract space obtained by completion can be "very large," however, this is not an issue when using finite dimensional approximations.

Let us denote by $\widehat{\Lambda}^{D}$ the completion of $\left(\boldsymbol{\Lambda}^{D},\|\cdot\|_{*}\right)$. Obviously, if $\left(\boldsymbol{\Lambda}^{D},\|\cdot\|_{*}\right)$ is complete, then it holds $\widehat{\Lambda}^{D}=\boldsymbol{\Lambda}^{D}$.

THEOREM 4.2. The minimization problem

$$
\inf _{\boldsymbol{\lambda} \in \widehat{\boldsymbol{\Lambda}}^{D}} J_{C, \mathcal{H}}(\boldsymbol{\lambda})
$$

has a unique solution $\boldsymbol{\lambda} \in \widehat{\boldsymbol{\Lambda}}^{D}$ satisfying

$$
\left(\widehat{\boldsymbol{\Lambda}}^{D}\right)^{\prime}\left\langle J_{C, \mathcal{H}}^{\prime}(\boldsymbol{\lambda}), \boldsymbol{\mu}\right\rangle_{\widehat{\boldsymbol{\Lambda}}^{D}}=\left(C \mathbf{u}^{\boldsymbol{\lambda}, f}, C \mathbf{u}^{\boldsymbol{\mu}}\right)_{\mathcal{H}}=0 \quad \forall \boldsymbol{\mu} \in \widehat{\boldsymbol{\Lambda}}^{D} .
$$

Proof. First, let us suppose that $\boldsymbol{\Lambda}^{D}$ is complete. For any $\boldsymbol{\lambda} \in \boldsymbol{\Lambda}^{D}$ we set

$$
\pi(\boldsymbol{\lambda}, \boldsymbol{\mu})={ }_{2}^{1}\left(C \mathbf{u}^{\boldsymbol{\lambda}}, C \mathbf{u}^{\boldsymbol{\mu}}\right)_{\mathcal{H}}, \quad \mathcal{L}(\boldsymbol{\mu})=-{ }_{2}^{1}\left(C \mathbf{u}^{\mathbf{0}, f}, C \mathbf{u}^{\boldsymbol{\mu}}\right)_{\mathcal{H}},
$$

so that $J_{C, \mathcal{H}}(\boldsymbol{\lambda})=\pi(\boldsymbol{\lambda}, \boldsymbol{\lambda})-2 \mathcal{L}(\boldsymbol{\lambda})+{ }_{2}^{1}\left\|C \mathbf{u}^{\mathbf{0}, f}\right\|_{\mathcal{H}}^{2}$ (see (4.2)). $\pi: \boldsymbol{\Lambda}^{D} \times \boldsymbol{\Lambda}^{D} \rightarrow \mathbb{R}$ is a bilinear symmetric form and thanks to Lemma 4.1 it is continuous and coercive with respect to the norm $\|\cdot\|_{*}$, while $\mathcal{L}: \Lambda^{D} \rightarrow \mathbb{R}$ is a linear continuous functional. Moreover $\left(\boldsymbol{\Lambda}^{D},\|\cdot\|_{*}\right)$ is a Hilbert space. By applying classical results of the calculus of variation (see, e.g., [15, Thm. I.1.1]), both existence and uniqueness of the solution follow. The Euler-Lagrange equation (4.4) follows by observing that $\boldsymbol{\Lambda}^{D^{\prime}}\left\langle J_{C, \mathcal{H}}^{\prime}(\boldsymbol{\lambda}), \boldsymbol{\mu}\right\rangle_{\boldsymbol{\Lambda}^{D}}=2 \pi(\boldsymbol{\lambda}, \boldsymbol{\mu})-2 \mathcal{L}(\boldsymbol{\mu})$, for any $\boldsymbol{\lambda}, \boldsymbol{\mu} \in \boldsymbol{\Lambda}^{D}$.

When $\Lambda^{D}$ is not complete the bilinear form $\pi$ (the linear functional $\mathcal{L}$, resp.) is continuous on $\boldsymbol{\Lambda}^{D}$ and it can be extended in a unique way to a continuous form (functional, resp.) on $\widehat{\Lambda}^{D}$ thanks to the Hahn-Banach theorem. The thesis follows by using the same arguments as before.

Remark 4.1. Other cost functionals could be defined, such as

$$
J_{1 / 2}\left(\lambda_{1}, \lambda_{2}\right)={ }_{2}^{1}\left\|u_{1}\left(\lambda_{1}\right)-u_{2}\left(\lambda_{2}\right)\right\|_{H_{00}^{1 / 2}\left(\Gamma_{1} \cup \Gamma_{2}\right)}^{2}
$$


and

$$
J_{-1 / 2}\left(\lambda_{1}, \lambda_{2}\right)={ }_{2}^{1}\left\|\partial_{n_{L}} u_{1}\left(\lambda_{1}\right)-\partial_{n_{L}} u_{2}\left(\lambda_{2}\right)\right\|_{\left(H_{00}^{1 / 2}\left(\Gamma_{1} \cup \Gamma_{2}\right)\right)^{\prime}}^{2} .
$$

By defining suitable inner products based on Steklov-Poincaré operators (see [19]) in both $H_{00}^{1 / 2}\left(\Gamma_{1} \cup \Gamma_{2}\right)$ and $\left(H_{00}^{1 / 2}\left(\Gamma_{1} \cup \Gamma_{2}\right)\right)^{\prime}$, it is possible to prove that both minimization problems inf $J_{1 / 2}(\boldsymbol{\lambda})$ and inf $J_{-1 / 2}(\boldsymbol{\lambda})$ are equivalent to inf $J_{1}(\boldsymbol{\lambda})$. Nevertheless we will not consider them in practice since they are quite expensive. Indeed, they would require the solution of additive boundary value problems involving boundary differential operators such as Laplace-Beltrami's (see [4]).

4.1. The optimality system. For the solution of the minimization problem (4.3) we apply the classical theory of optimal control problems (see [15]), where $\boldsymbol{\lambda} \in$ $\widehat{\Lambda}^{D}$ is the control, $\mathbf{u}^{\boldsymbol{\lambda}, f} \in \mathbf{V}$ is the state, $\mathcal{H}$ is the observation space, and $C: \mathbf{V} \rightarrow \mathcal{H}$ is the observation operator.

The numerical solution of the minimization problems (3.5) and (3.6) is achieved by solving (numerically) the optimality system (OS) associated with the Euler-Lagrange equation (4.4). The observation is distributed on the overlap (Cases 1-2) and the OS associated with the Euler-Lagrange equation (4.4) reads as follows: find $\boldsymbol{\lambda} \in \widehat{\boldsymbol{\Lambda}}^{D}$, $\mathbf{u} \in \mathbf{V}, \mathbf{p} \in \mathbf{V}^{D}$ such that

$$
\begin{aligned}
a_{i}\left(u_{i}, v_{i}\right) & =F_{i}\left(v_{i}\right), \quad u_{i}=\lambda_{i} \text { on } \Gamma_{i} & & \forall v_{i} \in V_{i}^{D}, \quad i=1,2, \\
a_{i}\left(v_{i}, p_{i}\right) & =(-1)^{i+1}\left(C \mathbf{u}, v_{i}\right)_{\mathcal{H}} & & \forall v_{i} \in V_{i}^{D}, \quad i=1,2, \\
\left(C \mathbf{u}, C \mathbf{u}^{\boldsymbol{\mu}}\right)_{\mathcal{H}} & =-\int_{\Gamma_{1}} \partial_{n_{L^{*}}} p_{1} \mu_{1} d \Gamma-\int_{\Gamma_{2}} \partial_{n_{L^{*}}} p_{2} \mu_{2} d \Gamma=0 & & \forall \boldsymbol{\mu}=\left(\mu_{1}, \mu_{2}\right) \in \widehat{\boldsymbol{\Lambda}}^{D},
\end{aligned}
$$

where the inner product on the observation space $\mathcal{H}$ is the canonical one. Note that the solution $\mathbf{u}$ of $(4.8)_{1}$ is in fact $\mathbf{u}^{\boldsymbol{\lambda}, f}$.

The following paragraphs are devoted to the derivation of both adjoint equations $(4.8)_{2}$ and the Euler-Lagrange equations $(4.8)_{3}$ for the Cases 1-2.

Case 1. Given $\left(u_{1}-u_{2}\right) \in H^{1}\left(\Omega_{12}\right)$ and denoting by $\chi_{12}$ the characteristic function associated with the open set $\Omega_{12}$, the adjoint problems read for $i=1,2$, find $p_{i} \in V_{i}^{D}$ :

$$
a_{i}\left(v_{i}, p_{i}\right)=(-1)^{i+1} \int_{\Omega_{i}} \chi_{12}\left(u_{1}-u_{2}\right) v_{i} d \Omega \quad \forall v_{i} \in V_{i}^{D},
$$

or alternatively,

$$
\begin{aligned}
L^{*} p_{i} & =(-1)^{i+1} \chi_{12}\left(u_{1}-u_{2}\right) & & \text { a.e. in } \Omega_{i}, \\
p_{i} & =0 & & \text { on } \partial \Omega_{i},
\end{aligned}
$$

where $L^{*}$ is the adjoint operator of $L$. Notice that $(4.9)_{1}$ holds a.e. in $\Omega_{i}$ by regularity results on elliptic problems which ensure that $p_{i} \in H^{2}\left(\Omega_{i}\right)$. The Euler-Lagrange equation (4.8) ${ }_{3}$ is derived in the following way (by using (4.9), (3.3), and integration by parts):

$$
\begin{aligned}
\left(C \mathbf{u}, C \mathbf{u}^{\boldsymbol{\mu}}\right)_{\mathcal{H}} & =\int_{\Omega_{12}}\left(u_{1}-u_{2}\right)\left(u_{1}^{\mu_{1}}-u_{2}^{\mu_{2}}\right) d \Omega=\sum_{i=1}^{2} \int_{\Omega_{i}} L^{*} p_{i} u_{i}^{\mu_{i}} d \Omega \\
& =\sum_{i=1}^{2}\left[-\int_{\Omega_{i}} L u_{i}^{\mu_{i}} p_{i} d \Omega-\int_{\partial \Omega_{i}} \partial_{n_{L^{*}}} p_{i} u_{i}^{\mu_{i}} d \Gamma+\int_{\partial \Omega_{i}} \partial_{n_{L}} u_{i}^{\mu_{i}} p_{i} d \Gamma\right] \\
& =-\sum_{i=1}^{2} \int_{\Gamma_{i}} \partial_{n_{L^{*}}} p_{i} \mu_{i} d \Gamma .
\end{aligned}
$$


Case 2. By definition of the inner product in $\mathcal{H}=H^{1}\left(\Omega_{12}\right)$, the adjoint problems read for $i=1,2$, find $p_{i} \in V_{i}^{D}$ :

$$
a_{i}\left(v_{i}, p_{i}\right)=(-1)^{i+1}\left[\int_{\Omega_{12}} \nabla\left(u_{1}-u_{2}\right) \cdot \nabla v_{i} d \Omega+\int_{\Omega_{12}}\left(u_{1}-u_{2}\right) v_{i} d \Omega\right] \quad \forall v_{i} \in V_{i}^{D}
$$

By introducing the linear and continuous functionals $G_{i}: V_{i} \rightarrow \mathbb{R}$,

$$
G_{i}\left(v_{i}\right)=(-1)^{i+1}\left[\int_{\Omega_{12}}\left(-\nabla \cdot\left(\nabla\left(u_{1}-u_{2}\right)\right)+\left(u_{1}-u_{2}\right)\right) v_{i} d \Omega+\int_{\Gamma_{1} \cup \Gamma_{2}} \partial\left(u_{1}-u_{2}\right) v_{i} d \Gamma\right],
$$

problems (4.10) also read

$$
\begin{array}{ll}
L^{*} p_{i}=G_{i} & \text { in } V_{i}^{\prime}, \\
p_{i}=0 & \text { on } \partial \Omega_{i},
\end{array}
$$

so that

$$
\begin{aligned}
\left(C \mathbf{u}, C \mathbf{u}^{\boldsymbol{\mu}}\right)_{H^{1}\left(\Omega_{12}\right)} & =\sum_{i=1}^{2}(-1)^{i+1}\left[\int_{\Omega_{12}} \nabla\left(u_{1}-u_{2}\right) \cdot \nabla u_{i}^{\mu_{i}} d \Omega+\int_{\Omega_{12}}\left(u_{1}-u_{2}\right) u_{i}^{\mu_{i}} d \Omega\right] \\
& =\sum_{i=1}^{2} G_{i}\left(u_{i}^{\mu_{i}}\right)=\sum_{i=1}^{2} \int_{\Omega_{i}} L^{*} p_{i} u_{i}^{\mu_{i}} d \Omega \\
& =\sum_{i=1}^{2}\left[-\int_{\Omega_{i}} L u_{i}^{\mu_{i}} p_{i} d \Omega-\int_{\partial \Omega_{i}} \partial_{n_{L^{*}}} p_{i} u_{i}^{\mu_{i}} d \Gamma+\int_{\partial \Omega_{i}} \partial_{n_{L}} u_{i}^{\mu_{i}} p_{i} d \Gamma\right] \\
& =-\sum_{i=1}^{2} \int_{\Gamma_{i}} \partial_{n_{L^{*}}} p_{i} \mu_{i} d \Gamma .
\end{aligned}
$$

Different considerations have to be taken into account when the observation is on the interfaces (Case 3). The Euler-Lagrange equation reads

$$
\left(\widehat{\boldsymbol{\Lambda}}^{D}\right)^{\prime}\left\langle J_{0, \Gamma}^{\prime}(\boldsymbol{\lambda}), \boldsymbol{\mu}\right\rangle_{\widehat{\boldsymbol{\Lambda}}^{D}}=\int_{\Gamma_{1} \cup \Gamma_{2}}\left(u_{1}^{\lambda_{1}, f}-u_{2}^{\lambda_{2}, f}\right)\left(u_{1}^{\mu_{1}}-u_{2}^{\mu_{2}}\right) d \Gamma=0 \quad \forall \boldsymbol{\lambda}, \boldsymbol{\mu} \in \widehat{\boldsymbol{\Lambda}}^{D},
$$

where $u_{i}^{\lambda_{i}, f}$ and $u_{i}^{\mu_{i}}$ are the solutions of (3.3) with nonnull and null $f$, respectively. The next theorem, Theorem 4.3, ensures that minimizing the cost functional (3.7) (or solving the Euler-Lagrange equation (4.13)) is equivalent to solving the following pseudooptimality system:

find $\mathbf{u}, \mathbf{p} \in \mathbf{V}^{D}, \boldsymbol{\lambda} \in \widehat{\boldsymbol{\Lambda}}^{D}$ such that

$$
\begin{array}{ll}
a_{i}\left(u_{i}, v_{i}\right)=F_{i}\left(v_{i}\right), \quad u_{i}=\lambda_{i} \text { on } \Gamma_{i} & \forall v_{i} \in V_{i}^{D}, \quad i=1,2, \\
a_{i}\left(p_{i}, v_{i}\right)=0, \quad p_{i}=(-1)^{i+1}\left(u_{1}-u_{2}\right) \text { on } \Gamma_{i} & \forall v_{i} \in V_{i}^{D}, \quad i=1,2, \\
\int_{\Gamma_{1}}\left(\left(u_{1}-u_{2}\right)+p_{2}\right) \mu_{1} d \Gamma+\int_{\Gamma_{2}}\left(-\left(u_{1}-u_{2}\right)+p_{1}\right) \mu_{2} d \Gamma=0 & \forall \boldsymbol{\mu} \in \widehat{\boldsymbol{\Lambda}}^{D} .
\end{array}
$$

Note that the solution $\mathbf{u}$ of $(4.14)_{1}$ is in fact $\mathbf{u}^{\boldsymbol{\lambda}, f}$. 
Remark 4.2. We use the adjective pseudo to mean that (4.14) is derived by a different approach than a classical optimality system. In fact, $(4.14)_{2}$ differs from the dual state equation and $(4.14)_{3}$ is not obtained by an integration by parts procedure.

THEOREM 4.3. The system (4.14) has a unique solution whose control component $\boldsymbol{\lambda}$ is the solution of (4.3) (or equivalently (4.13)).

Proof. Existence. Let $\boldsymbol{\lambda}$ be the solution of

$$
\inf _{\boldsymbol{\lambda} \in \widehat{\boldsymbol{\Lambda}}^{D}} J_{0, \Gamma}(\boldsymbol{\lambda})
$$

(that exists and is unique in view of Theorem 4.2), then it is a solution of (4.14). As a matter of fact, if $\boldsymbol{\lambda}$ is the solution of (4.15), then it satisfies (4.13), $u_{1}^{\lambda_{1}, f}-u_{2}^{\lambda_{2}, f}=0$ on $\Gamma_{1} \cup \Gamma_{2}$ and the solutions $p_{i}$ of (4.14) $)_{2}$ are null in $\Omega_{i}$ for $i=1,2$. Therefore (4.14) is satisfied and (4.14) admits at least one solution.

Uniqueness. Let us start with the case $f=0$. We define the operator $\chi: \boldsymbol{\Lambda}^{D} \rightarrow$ $\left(\Lambda^{D}\right)^{\prime}$

$$
\left(\boldsymbol{\Lambda}^{D}\right)^{\prime}\langle\chi(\boldsymbol{\lambda}), \boldsymbol{\mu}\rangle_{\boldsymbol{\Lambda}^{D}}=\int_{\Gamma_{1}}\left(\left(u_{1}^{\lambda_{1}}-u_{2}^{\lambda_{2}}\right)+p_{2}^{\boldsymbol{\lambda}}\right) \mu_{1} d \Gamma+\int_{\Gamma_{2}}\left(-\left(u_{1}^{\lambda_{1}}-u_{2}^{\lambda_{2}}\right)+p_{1}^{\boldsymbol{\lambda}}\right) \mu_{2} d \Gamma
$$

where $u_{i}^{\lambda_{i}}$ and $p_{i}^{\boldsymbol{\lambda}}$ (for $\left.i=1,2\right)$ are the solutions of $(4.14)_{1,2}$ with $f=0$. The linearity of the operator $\chi: \boldsymbol{\lambda} \mapsto \chi(\boldsymbol{\lambda})$, follows from that of $L$. It remains to prove that $\operatorname{ker}(\chi)=\{\mathbf{0}\}$.

In view of $(4.14)_{2}, p_{1}^{\lambda} \in V_{1}$ and $p_{2}^{\boldsymbol{\lambda}} \in V_{2}$; therefore, $\left.p_{i}^{\boldsymbol{\lambda}}\right|_{\Gamma_{i}} \in H_{00}^{1 / 2}\left(\Gamma_{i}\right)$ and, if $\lambda \in \operatorname{ker}(\chi)$, by (4.16) it holds

$$
p_{2}^{\lambda}=-\left(u_{1}^{\lambda_{1}}-u_{2}^{\lambda_{2}}\right) \quad \text { on } \Gamma_{1}, \quad p_{1}^{\lambda}=u_{1}^{\lambda_{1}}-u_{2}^{\lambda_{2}} \quad \text { on } \Gamma_{2} .
$$

It follows that $p_{1}^{\boldsymbol{\lambda}}$ and $p_{2}^{\boldsymbol{\lambda}}$ satisfy the system

$$
\begin{array}{lll}
a_{1}\left(p_{1}^{\boldsymbol{\lambda}}, v_{1}\right)=0 & \forall v_{1} \in H_{0}^{1}\left(\Omega_{1}\right), & p_{1}^{\boldsymbol{\lambda}}=-p_{2}^{\boldsymbol{\lambda}} \text { on } \Gamma_{1}, \\
a_{2}\left(p_{2}^{\boldsymbol{\lambda}}, v_{2}\right)=0 & \forall v_{2} \in H_{0}^{1}\left(\Omega_{2}\right), & p_{2}^{\boldsymbol{\lambda}}=-p_{1}^{\boldsymbol{\lambda}} \text { on } \Gamma_{2} .
\end{array}
$$

By using the same arguments as in the proof of Proposition 2.1, we define $w=$ $p_{1}^{\lambda}+p_{2}^{\lambda} \in H^{1}\left(\Omega_{12}\right)$ and, thanks to (4.18), $w \in H_{0}^{1}\left(\Omega_{12}\right), L w=0$ a.e. in $\Omega_{12}$ and then $w \equiv 0$ in $\Omega_{12}$. If we set

$$
p= \begin{cases}p_{1}^{\lambda} & \text { in } \Omega_{1} \backslash \Omega_{12}, \\ p_{1}^{\lambda}=-p_{2}^{\lambda} & \text { in } \Omega_{12}, \\ -p_{2}^{\lambda} & \text { in } \Omega_{2} \backslash \Omega_{12},\end{cases}
$$

then $p \in H_{0}^{1}(\Omega)$ and it satisfies $a(p, v)=0 \forall v \in H_{0}^{1}(\Omega)$, yielding $p \equiv 0$ in $\Omega$, i.e., $p_{1}^{\lambda}=0$ in $\Omega_{1}$ and $p_{2}^{\lambda}=0$ in $\Omega_{2}$. It follows that $u_{1}^{\lambda_{1}}-u_{2}^{\lambda_{2}}=0$ on $\Gamma_{1} \cup \Gamma_{2}$.

By invoking again the same arguments, however, now on the primal equations $(4.14)_{1}$ with $f=0$, it holds $u_{i}^{\lambda_{i}}=0$ in $\Omega_{i}$ and then $\lambda_{i}=0$ on $\Gamma_{i}$, for $i=1,2$. By a density argument the uniqueness is proven in $\widehat{\Lambda}^{D}$.

When $f \neq 0$, for $i=1,2$ let $p_{i}^{f} \in V_{i}$ denote the solution of

$$
a\left(p_{i}^{f}, v_{i}\right)=0 \quad \forall v_{i} \in V_{i}, \quad p_{i}^{f}=(-1)^{i+1}\left(u_{1}^{0, f}-u_{2}^{0, f}\right) \quad \text { on } \Gamma_{i} .
$$

The pseudooptimality system (4.14) can be rewritten as

$$
\left(\boldsymbol{\Lambda}^{D}\right)^{\prime}\langle\chi(\boldsymbol{\lambda}), \boldsymbol{\mu}\rangle_{\boldsymbol{\Lambda}^{D}}=-{ }_{\left(\boldsymbol{\Lambda}^{D}\right)^{\prime}}\left\langle\mathcal{A}_{f}, \boldsymbol{\mu}\right\rangle_{\boldsymbol{\Lambda}^{D}} \quad \forall \boldsymbol{\mu} \in \boldsymbol{\Lambda}^{D},
$$


where $\mathcal{A}_{f}: \boldsymbol{\Lambda}^{D} \rightarrow\left(\boldsymbol{\Lambda}^{D}\right)^{\prime}$ is defined by

$$
{ }_{\left(\boldsymbol{\Lambda}^{D}\right)^{\prime}}\left\langle\mathcal{A}_{f}, \boldsymbol{\mu}\right\rangle_{\boldsymbol{\Lambda}^{D}}=\int_{\Gamma_{1}}\left(\left(u_{1}^{0, f}-u_{2}^{0, f}\right)+p_{2}^{f}\right) \mu_{1} d \Gamma+\int_{\Gamma_{1}}\left(-\left(u_{1}^{0, f}-u_{2}^{0, f}\right)+p_{1}^{f}\right) \mu_{2} d \Gamma .
$$

The thesis follows by applying the same arguments as before.

Remark 4.3. Solving the optimality system (4.14) is very attractive since both problems with unknowns $u_{i}$ and $p_{i}$ are of the same nature (no adjoint equations are needed here, contrary to what happens in (4.8)); moreover, only zeroth order (Dirichlet) traces are required and no flux has to be computed on the interfaces.

When the boundary value problems are discretized by a Galerkin method (e.g., finite element methods (FEM) or spectral element methods (SEM)), the discretizations in $\Omega_{1}$ and $\Omega_{2}$ may be totally unrelated: the two grids used inside each subdomain $\Omega_{i}$, and/or the local polynomial degrees, may differ from one another. If the grids do not match on $\Omega_{12}$, the term $\left(u_{1}^{\lambda_{1}}-u_{2}^{\lambda_{2}}\right)$ can be computed through interpolation operators (from the mesh in $\Omega_{1}$ to that in $\Omega_{2}$ or vice versa). When distributed observation is considered (as in minimizing $J_{0}$ and $J_{1}$ ) the interpolation step could be very expensive if the overlapping region is wide and the meshes are very fine in $\Omega_{12}$, unless matching meshes on the overlap are taken into account.

Different conclusions can be reached when interface observation is considered (as in $\left.J_{0, \Gamma}\right)$ since, in such cases, the interpolation is required only on the interfaces $\Gamma_{i}$, with a computational cost that does not affect the global efficiency of the method.

Remark 4.4. The analysis carried out till now can be applied to decompositions of $\Omega$ in $M>2$ subdomains. In this respect, we distinguish between stripwise decompositions, in which each overlapped region is shared only by two subdomains, and crosswise decompositions, in which more than two subdomains can share a nonempty set. In the former case the subdomains $\Omega_{k}$ for $k=1, \ldots, M$ can be numbered sequentially, so that all the odd (even, resp.) subdomains can be grouped in a unique disconnected subdomain $\widetilde{\Omega}_{1}\left(\widetilde{\Omega}_{2}\right.$, resp. $)$ and the analysis presented above still holds provided $\Omega_{i}$ is replaced by $\widetilde{\Omega}_{i}$ (for $i=1,2$ ). Otherwise in the latter case, we define $\Omega_{i j}=\Omega_{i} \cap \Omega_{j}$ for $i \neq j$ and we replace the cost functionals of Table 4.1 by

$$
J_{\mathcal{H}}(\boldsymbol{\lambda})=\frac{1}{2} \sum_{\substack{i, j=1 \\ i>j \\ \Omega_{i j} \neq \emptyset}}^{M}\left\|u_{i}^{\lambda_{i}, f}-u_{j}^{\lambda_{j}, f}\right\|_{\mathcal{H}_{i j}}^{2}
$$

where $\mathcal{H}_{i j}$ is $L^{2}\left(\Omega_{i j}\right), H^{1}\left(\Omega_{i j}\right)$, or $L^{2}\left(\partial \Omega_{i j}\right)$. The formulation of both optimality systems (4.8) and (4.14) follows by replacing $\Omega_{12}$ with $\Omega_{i j}$ for any $i, j=1, \ldots, M$ and counting every overlap only once.

4.2. Analysis of the optimal control problems with Robin interface controls. We consider now the case of Robin controls at the interfaces with distributed observation. Like in the previous section, for the sake of simplicity we still assume that $\phi_{D}=0$ on $\partial \Omega=\Gamma_{D}$. We introduce the spaces $\Lambda^{R}=\Lambda_{1}^{R} \times \Lambda_{2}^{R}$ and

$$
\Lambda_{12}^{R}=\left(H_{00}^{1 / 2}\left(\Gamma_{1} \cup \Gamma_{2}\right)\right)^{\prime} .
$$

With a slight abuse of notation, we still indicate by $\mathbf{u}^{\boldsymbol{\lambda}, f}$ the solution of the control problem although now we are considering Robin interface controls instead of Dirichlet ones. More precisely, $\mathbf{u}^{\boldsymbol{\lambda}, f}$ is now the solution of (3.4). 
Notice that the characterizations of the cost functionals, as done in (4.1)-(4.2), still hold considering now $\boldsymbol{\Lambda}^{R}$ instead of $\boldsymbol{\Lambda}^{D}$ and $\Lambda_{12}^{R}$ instead of $\Lambda_{12}^{D}$. Moreover, we can prove the following results.

Lemma 4.4. For the Cases $1-2,\left\|C \mathbf{u}^{\boldsymbol{\lambda}}\right\|_{\mathcal{H}}$ is a norm on the control space $\mathbf{\Lambda}^{R}$.

THEOREM 4.5. For the Cases 1-2, the minimization problem

$$
\inf _{\boldsymbol{\lambda} \in \widehat{\boldsymbol{\Lambda}}^{R}} J_{C, \mathcal{H}}(\boldsymbol{\lambda})
$$

has a unique solution $\boldsymbol{\lambda} \in \widehat{\boldsymbol{\Lambda}}^{R}$ satisfying

$$
\left(\widehat{\boldsymbol{\Lambda}}^{R}\right)^{\prime}\left\langle J_{C, \mathcal{H}}^{\prime}(\boldsymbol{\lambda}), \boldsymbol{\mu}\right\rangle_{\widehat{\boldsymbol{\Lambda}}^{R}}=\left(C \mathbf{u}^{\boldsymbol{\lambda}, f}, C \mathbf{u}^{\boldsymbol{\mu}}\right)_{\mathcal{H}}=0 \quad \forall \boldsymbol{\mu} \in \widehat{\boldsymbol{\Lambda}}^{R},
$$

where $\widehat{\Lambda}^{R}$ is the completion of $\boldsymbol{\Lambda}^{R}$ with respect to the norm $\|\cdot\|_{\mathcal{H}}$.

The proofs of these results follow the ones of Lemma 4.1 and Theorem 4.2, respectively.

Using Robin controls at the interfaces, the Euler-Lagrange equation (4.22) for both Cases 1 and 2 leads to the following optimality system: find $\boldsymbol{\lambda} \in \widehat{\boldsymbol{\Lambda}}^{R}, \mathbf{u} \in \mathbf{V}$, $\mathbf{p} \in \mathbf{V}$ such that

$$
\begin{aligned}
a_{i}\left(u_{i}, v_{i}\right)+\int_{\Gamma_{i}} \beta u_{i} v_{i} & =F_{i}\left(v_{i}\right)+\int_{\Gamma_{i}} \lambda_{i} v_{i} & & \forall v_{i} \in V_{i}, \quad i=1,2, \\
a_{i}\left(v_{i}, p_{i}\right)+\int_{\Gamma_{i}} \beta v_{i} p_{i} & =(-1)^{i+1}\left(C \mathbf{u}, v_{i}\right)_{\mathcal{H}} & & \forall v_{i} \in V_{i}, \quad i=1,2, \\
\left(C \mathbf{u}, C \mathbf{u}^{\boldsymbol{\mu}}\right)_{\mathcal{H}} & =\int_{\Gamma_{1}} p_{1} \mu_{1} d \Gamma+\int_{\Gamma_{2}} p_{2} \mu_{2} d \Gamma=0 & & \forall \boldsymbol{\mu}=\left(\mu_{1}, \mu_{2}\right) \in \widehat{\boldsymbol{\Lambda}}^{R} .
\end{aligned}
$$

Note that the solution $\mathbf{u}$ of $(4.23)_{1}$ is in fact $\mathbf{u}^{\boldsymbol{\lambda}, f}$.

The characterization of the right-hand side done for the case of Dirichlet controls still applies here.

5. ICDD for heterogeneous problems. We can take inspiration from the previous developments to address heterogeneous and multiphysics problems by a similar approach. For the sake of exposition we consider two examples: the coupling between advection and advection-diffusion equations and the associated ICDD formulation for the case with interface observation, and a Stokes-Darcy coupled problem to model the filtration of fluids through porous media. The latter problem will be fully addressed in [7], where the associated ICDD formulation will be analyzed and numerical results discussed.

5.1. ICDD with interface observation for the heterogeneous A-AD coupling. Let us consider the coupling of advection and advection-diffusion equations (in brief A-AD), that is of interest when the advection field dominates over the diffusion and the solution of the global advection-diffusion problem features a boundary layer. In such a case the presence of the viscous term is undoubtable in the subregion adjacent to the layer, but at the same time it is negligible far from the layer. A preliminary study of the A-AD coupling with overlapping subdomains has been carried out in $[12,1,4]$.

The same notations introduced above are used here; therefore we look for two functions $u_{1}$ and $u_{2}$ (defined in $\Omega_{1}$ and $\Omega_{2}$, resp.) such that $u_{1}$ satisfies the advectionreaction equation

$$
L_{1} u_{1}=\operatorname{div}\left(\mathbf{b} u_{1}\right)+b_{0} u_{1}=f \quad \text { in } \Omega_{1},
$$




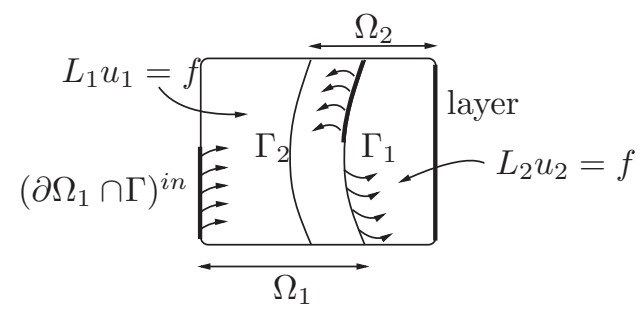

FIG. 5.1. Graphic representation of a 2D A-AD heterogeneous coupling.

while $u_{2}$ satisfies the advection-diffusion-reaction equation

$$
L_{2} u_{2}=\operatorname{div}\left(-K \nabla u_{2}+\mathbf{b} u_{2}\right)+b_{0} u_{2}=f \quad \text { in } \Omega_{2} .
$$

For any nonempty subset $S \subseteq \partial \Omega_{1}$, we set

$$
\begin{aligned}
\text { the inflow part of } S: & S^{\text {in }}=\{\mathbf{x} \in S: \mathbf{b}(\mathbf{x}) \cdot \mathbf{n}(\mathbf{x})<0\}, \\
\text { the outflow part of } S: & S^{\text {out }}=\{\mathbf{x} \in S: \mathbf{b}(\mathbf{x}) \cdot \mathbf{n}(\mathbf{x})>0\}
\end{aligned}
$$

and $S^{0}=S \backslash\left(S^{\text {in }} \cup S^{\text {out }}\right)$.

The boundary conditions for problem (5.1) are assigned on the inflow boundary $\left(\partial \Omega_{1}\right)^{i n}$; for simplicity we set $u_{1}=0$ on the external boundary $\left(\partial \Omega_{1} \backslash \Gamma_{1}\right)^{i n}$ and $u_{1}=u_{2}$ on $\Gamma_{1}^{i n}$ (see Figure 5.1). Assuming that meas $\left(\Gamma_{2}^{0}\right)=0$, we assign homogeneous Dirichlet boundary conditions $u_{2}=0$ for problem (5.2) on the external boundary $\partial \Omega_{2} \backslash \Gamma_{2}$, and $u_{2}=u_{1}$ on $\Gamma_{2}$.

We notice that in general there is no guarantee that $u_{1}=u_{2}$ in $\Omega_{12}$ (see, e.g., $[12,1])$.

When the computational domain is partitioned into two nonoverlapping subdomains with sharp interface, the heterogeneous A-AD coupling has been analyzed in $[11,10]$, where a suitable set of interface conditions has been provided expressing the continuity of the velocity field across the inflow part of the unique interface $\Gamma$ and the continuity of the fluxes across the whole interface $\Gamma$.

In order to describe ICDD method for A-AD coupling, we first define the Hilbert space (see $[11,10])$

$$
L_{\mathbf{b}}^{2}\left(\Gamma_{1}^{i n}\right)=\left\{v: \Gamma_{1}^{i n} \rightarrow \mathbb{R}:\left(\left|\mathbf{b} \cdot \mathbf{n}_{\Gamma_{1}^{i n}}\right|\right)^{1 / 2} v \in L^{2}\left(\Gamma_{1}^{i n}\right)\right\} .
$$

The ICDD formulation for the heterogeneous coupling (5.1)-(5.2) reads as follows (see Figure 5.2): look for the interface controls $\lambda_{1} \in L_{\mathbf{b}}^{2}\left(\Gamma_{1}^{i n}\right)$ and $\lambda_{2} \in H_{00}^{1 / 2}\left(\Gamma_{2}\right)$ solutions of

$$
\inf _{\lambda_{1}, \lambda_{2}}\left[J_{\mathbf{b}}\left(\lambda_{1}, \lambda_{2}\right)=\frac{1}{2} \int_{\Gamma_{1}^{i n} \cup \Gamma_{2}}|\mathbf{b} \cdot \mathbf{n}|\left(u_{1}^{\lambda_{1}, f}-u_{2}^{\lambda_{2}, f}\right)^{2}\right],
$$

where $u_{1}^{\lambda_{1}, f}$ and $u_{2}^{\lambda_{2}, f}$ are the solutions of

$$
\left\{\begin{array} { l l } 
{ L _ { 1 } u _ { 1 } ^ { \lambda _ { 1 } , f } = f } & { \text { in } \Omega _ { 1 } , } \\
{ u _ { 1 } ^ { \lambda _ { 1 } , f } = 0 } & { \text { on } ( \partial \Omega _ { 1 } \backslash \Gamma _ { 1 } ) ^ { i n } , } \\
{ u _ { 1 } ^ { \lambda _ { 1 } , f } = \lambda _ { 1 } } & { \text { on } \Gamma _ { 1 } ^ { i n } }
\end{array} \quad \left\{\begin{array}{ll}
L_{2} u_{2}^{\lambda_{2}, f}=f & \text { in } \Omega_{2}, \\
u_{2}^{\lambda_{2}, f}=0 & \text { on } \partial \Omega_{2} \backslash \Gamma_{2}, \\
u_{2}^{\lambda_{2}, f}=\lambda_{2} & \text { on } \Gamma_{2} .
\end{array}\right.\right.
$$




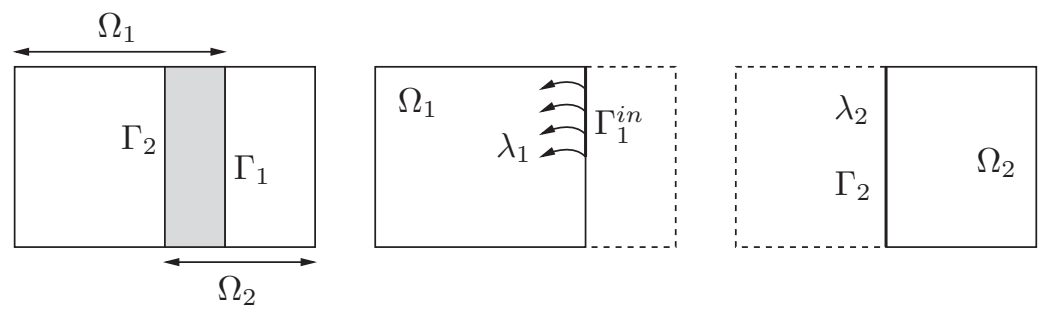

FIG. 5.2. Domain decomposition for the heterogeneous A-AD coupling.

Set

$$
\begin{aligned}
V_{1} & =\left\{v \in L^{2}\left(\Omega_{1}\right), \operatorname{div}(\mathbf{b} v) \in L^{2}\left(\Omega_{1}\right), v \in L_{\mathbf{b}}^{2}\left(\partial \Omega_{1}\right)\right\}, \\
V_{1}^{D} & =\left\{v \in V_{1}:\left.v\right|_{\left(\partial \Omega_{1} \backslash \Gamma_{1}\right)^{i n}}=0\right\}, \quad \Lambda_{1}^{D}=L_{\mathbf{b}}^{2}\left(\Gamma_{1}^{i n}\right),
\end{aligned}
$$

and take $V_{2}, V_{2}^{D}$, and $\Lambda_{2}^{D}$ as in section 2 ; therefore, define $\mathbf{V}=V_{1} \times V_{2}, \mathbf{V}^{D}=$ $V_{1}^{D} \times V_{2}^{D}$, and $\Lambda^{D}=\Lambda_{1}^{D} \times \Lambda_{2}^{D}$, and the bilinear forms

$$
\begin{aligned}
& a_{1}\left(u_{1}, v_{1}\right)=-\int_{\Omega_{1}}\left(\mathbf{b} u_{1}\right) \cdot \nabla v_{1}+\int_{\Omega_{1}} b_{0} u_{1} v_{1}+\int_{\partial \Omega_{1}^{\text {out }}} \mathbf{b} \cdot \mathbf{n} u_{1} v_{1}, \\
& a_{2}\left(u_{2}, v_{2}\right)=\int_{\Omega_{2}} K \nabla u_{2} \cdot \nabla v_{2}-\int_{\Omega_{2}}\left(\mathbf{b} u_{2}\right) \cdot \nabla v_{2}+\int_{\Omega_{2}} b_{0} u_{2} v_{2} .
\end{aligned}
$$

The A-AD counterpart of the optimality system (4.14), that is used in practice to solve the minimization problem, reads find $\mathbf{u}, \mathbf{p} \in \mathbf{V}, \boldsymbol{\lambda} \in \boldsymbol{\Lambda}^{D}$ such that

$$
\begin{array}{lll}
a_{1}\left(u_{1}, v_{1}\right)=\int_{\Omega_{1}} f v_{1}, & u_{1}=\lambda_{1} \text { on } \Gamma_{1}^{i n} & \forall v_{1} \in V_{1}^{D}, \\
a_{2}\left(u_{2}, v_{2}\right)=\int_{\Omega_{2}} f v_{2}, & u_{2}=\lambda_{2} \text { on } \Gamma_{2} & \forall v_{2} \in V_{2}^{D}, \\
a_{1}\left(p_{1}, v_{1}\right)=0, & p_{1}=u_{1}-u_{2} \text { on } \Gamma_{1}^{i n} & \forall v_{1} \in V_{1}^{D}, \\
a_{2}\left(p_{2}, v_{2}\right)=0, & p_{2}=u_{2}-u_{1} \text { on } \Gamma_{2} & \forall v_{2} \in V_{2}^{D}, \\
\int_{\Gamma_{1}^{i n}} \mathbf{b} \cdot \mathbf{n}\left(\left(u_{1}-u_{2}\right)+p_{2}\right) \mu_{1} d \Gamma+\int_{\Gamma_{2}} \mathbf{b} \cdot \mathbf{n}\left(\left(u_{2}-u_{1}\right)+p_{1}\right) \mu_{2} d \Gamma=0 & \forall \boldsymbol{\mu} \in \widehat{\boldsymbol{\Lambda}}^{D} .
\end{array}
$$

The numerical results of Test 3 in section 6 refer to the finite element approximation of (5.6)-(5.7). A more thorough investigation of the ICDD method for the coupled A-AD problem (5.1)-(5.2) is carried out in [6].

5.2. Heterogeneous Stokes-Darcy coupling. A second instance of a heterogeneous problem is provided by the coupled free/porous-media flow problem. The computational domain is a region naturally split into two parts: one, $\Omega_{1}$, occupied by the fluid, the other, $\Omega_{2}$, by the porous media. The fluid in $\Omega_{1}$ can filtrate through the adjacent porous medium. From the physical point of view, $\Gamma$ is a surface separating the two domains, but we can also suppose that there is a thin overlapping region of coexistence of both media (see Figure 5.3). We assume that the fluid domain has a fixed surface, i.e., we neglect here the case of free-surface flows. 

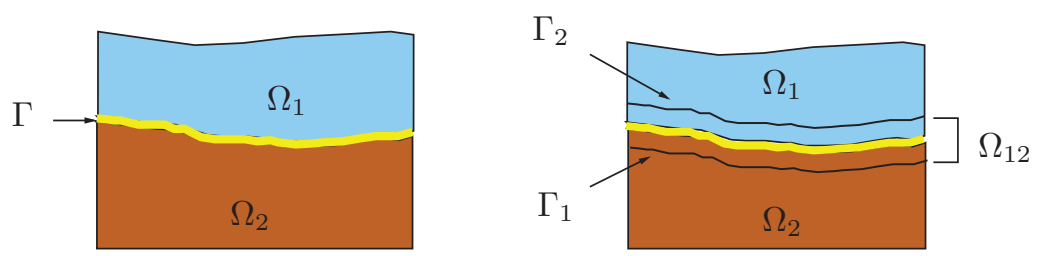

FIG. 5.3. Representation of a $2 D$ section of a possible computational domain for the coupled free/porous-media flow problem. At left, a decomposition with sharp interface $\Gamma$; at right, a decomposition with overlap.

Far from being exhaustive on the analysis of this problem (we refer to $[17,8$, 4] for its precise statement), here we briefly formulate the coupling for overlapping decompositions. The main advantage is to avoid using sharp interfaces which would require an in-depth knowledge of the local physical behavior (interface conditions) as the Beavers-Joseph-Saffman in the Stokes-Darcy coupling (see, e.g., [8]).

By using the Stokes equations for describing the motion of the fluid in $\Omega_{1}$ and Darcy's law for expressing the relation between velocity and pressure in porous media the heterogeneous problem can be formulated as follows.

Given $T>0$, a vector valued function $\mathbf{f}$ in $\Omega_{1} \times(0, T)$, a positive viscosity $\nu$, and a symmetric positive definite diagonal tensor $K=\left(K_{i j}\right)_{i, j=1, \ldots, d}$, we look for the fluid velocity $\mathbf{u}_{1}=\mathbf{u}_{1}(\mathbf{x}, t)$ and the fluid pressure $p_{1}=p_{1}(\mathbf{x}, t)$ in $\Omega_{1} \times(0, T)$, and for the piezometric head $\varphi=\varphi(\mathbf{x}, t)$ (that essentially represents the fluid pressure in the porus medium) in $\Omega_{2} \times(0, T)$, such that

$$
\begin{array}{ll}
\partial_{t} \mathbf{u}_{1}-\nu \Delta \mathbf{u}_{1}+\nabla p_{1}=\mathbf{f} & \text { in } \Omega_{1} \times(0, T), \\
\nabla \cdot \mathbf{u}_{1}=0 & \text { in } \Omega_{1} \times(0, T), \\
-\nabla \cdot(K \nabla \varphi)=0 & \text { in } \Omega_{2} \times(0, T), \\
\text { BC for } \mathbf{u}_{1} & \text { on }\left(\partial \Omega_{1} \backslash \Gamma_{1}\right) \times(0, T), \\
\text { BC for } \varphi & \text { on }\left(\partial \Omega_{2} \backslash \Gamma_{2}\right) \times(0, T), \\
\text { initial conditions for } \mathbf{u}_{1} & \text { in } \Omega_{1} \times\{0\},
\end{array}
$$

and finally we close the system by requiring that $\mathbf{u}_{1}$ and $K \nabla \varphi$ match in some sense in $\Omega_{12} \times(0, T)$ or on $\left(\Gamma_{1} \cup \Gamma_{2}\right) \times(0, T)$.

This problem will be more carefully addressed in [7]. Some preliminary results in this direction have been published in [20].

6. Numerical results. We give here a few numerical results referring to the solution of elliptic boundary value problems by ICDD and we refer to [6] for an indepth description of the discretization of the systems (4.8) and (4.14), as well as for efficiency and robustness analysis of the ICDD method.

The boundary value problems displayed in (4.14) are discretized by $h p$-FEM of either simplicial $\left(\mathbb{P}_{r}\right)$ or quadrilateral $\left(\mathbb{Q}_{p}\right)$ type. (In the latter case $h p$-FEM are also known as SEM; see [3] for details.) The associated linear system has a blockwise structure with unknowns $\left[u_{1}, u_{2}, p_{1}, p_{2}, \lambda_{1}, \lambda_{2}\right]^{t}$. Then we derive its Schur complement system with respect to the control variables $\lambda_{i}$ (see [6]), and we solve the latter by the Bi-CGSTAB method (see [23]).

At each iteration of the Bi-CGSTAB method two matrix-vector products between the Schur complement matrix and the vector of discrete interface controls have to be performed. Each product involves addressing the three steps of the optimality system (either (4.8) or (4.14)) for given interface control functions, that is 

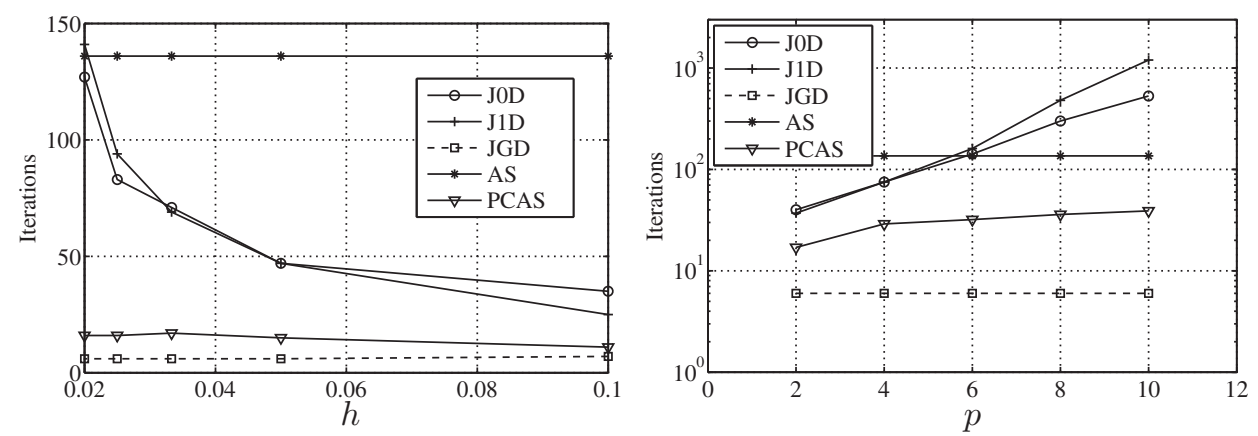

FIG. 6.1. Iterations number of ICDD and Schwarz methods: at left, versus the mesh size $h$ for $\mathbb{P}_{1}$ discretization; at right, versus the polynomial degree $p$ for $\mathbb{Q}_{p}$ discretization and $h=0.2$.

- solve $M$ primal state problems in parallel ( $M$ denotes the global number of subdomains);

- solve $M$ dual state problems in parallel;

- evaluate the interface contributions in the Euler-Lagrange equations.

One iteration of the ICDD method is in fact one Bi-CGSTAB iteration and, because the latter requires two matrix-vector operations, it costs the solution of $4 M$ local boundary value problems. The cost of one ICDD iteration is four times the cost of one iteration of the classic additive Schwarz (AS) method and it is less than twice the cost of one iteration of Bi-CGSTAB preconditioned by AS with coarse grid (PASC). The latter requires us to solve $2 M$ local boundary value problems at each iteration and 2 matrix-vector products for the global stiffness matrix on the fine grid. See, e.g., $[22,3]$.

In spite of that, the number of iterations required by ICDD with cost functional $J_{0, \Gamma}$ and Dirichlet controls is in general lower than that required by Schwarz methods.

As an example, let us consider the elliptic problem $(2.2)$ in $\Omega=(0,1)^{2}$, set $K=1, \gamma=0, \mathbf{b}=(0,0)$, and Dirichlet boundary conditions on $\partial \Omega$. The functions $f$ and $\phi_{D}$ are defined so that the exact solution of the differential problem (2.2) is $u(x, y)=\sin (\pi x y)+1$.

The domain $\Omega$ is decomposed into $2 \times 2$ subdomains of the same size, the interfaces $\Gamma_{i}$ (for $i=1, \ldots, 8$ ) are parallel to the $x$ and $y$ axes and symmetric with respect to the right lines passing at the midponts of the side of $\Omega$. The thickness $\delta_{12}$ of the overlaps is uniform and the local grids match on the overlaps.

In Figures 6.1 and 6.2 we compare the convergence rate of ICDD with Dirichlet controls with that of Schwarz methods under a common stopping criterion with tolerance $\epsilon=10^{-12}$. The number of iterations required by the ICDD method with Dirichlet controls and cost functionals $J_{0}, J_{1}$, and $J_{0, \Gamma}$ (named J0D, J1D and JGD, resp.) as well as the number of both AS and PASC are shown versus the mesh size $h$ for $\mathbb{P}_{1}$ discretization, the polynomial degree $p$ for $\mathbb{Q}_{p}$ discretization, the overlap thickness $\delta_{12}$ for both $\mathbb{P}_{1}$ and $\mathbb{Q}_{p}$.

The analysis of the condition number of the Schur complement matrix for the ICDD method, as well as the design of a suitable coarse correction that may guarantee scalability as for PASC, are in progress and will be presented in a future work ([5]). Nevertheless, preliminary results show that the convergence rate of ICDD with $J_{0, \Gamma}$ and Dirichlet controls is independent of the discretization parameters while it depends on the overlap thickness as $\delta_{12}^{-1 / 2}$ (see Figures 6.1 and 6.2). 

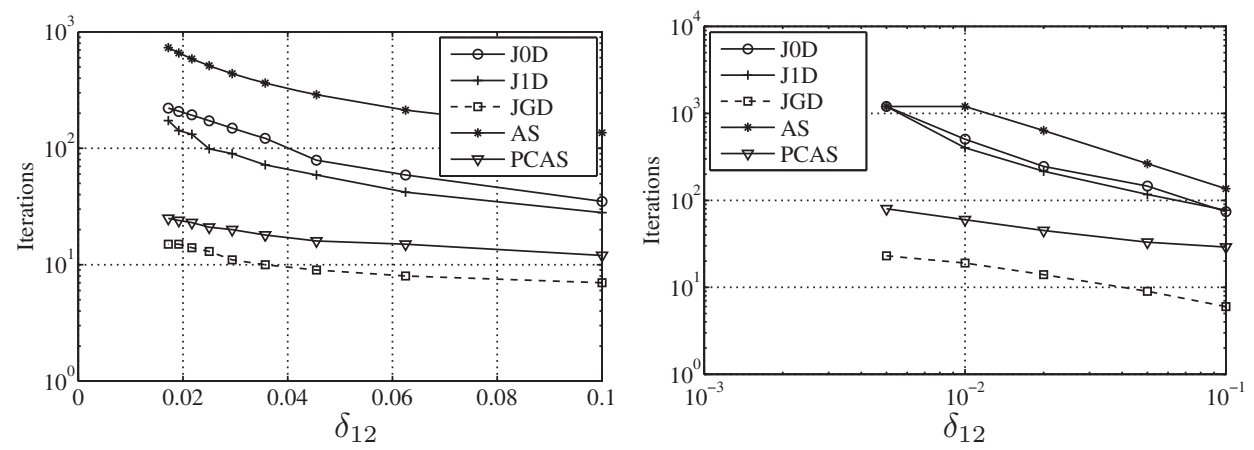

FIG. 6.2. Iterations number of ICDD and Schwarz methods versus the overlap thickness: at left, for $\mathbb{P}_{1}$ discretization and uniform $h=\delta_{12}$; at right, for $\mathbb{Q}_{4}$ discretization and nonuniform $h$.
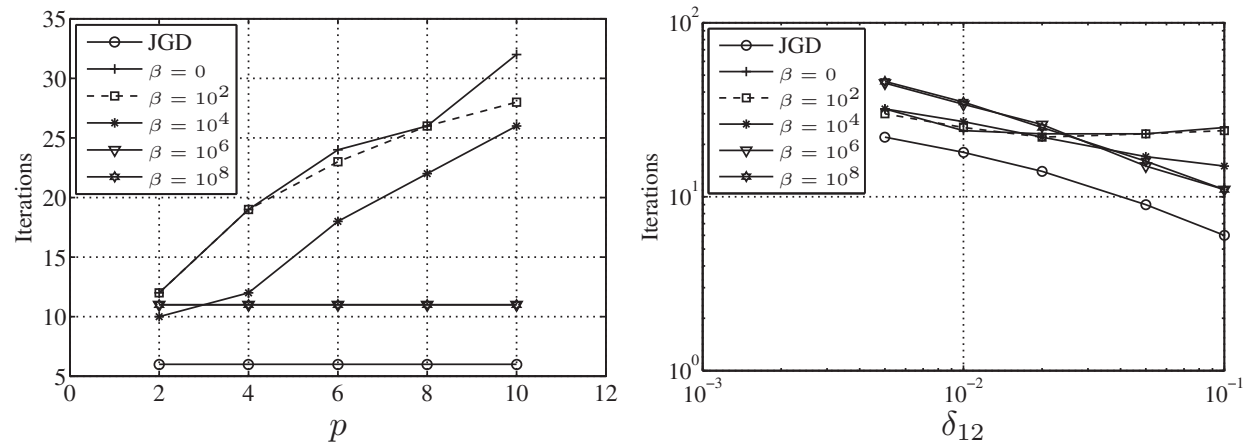

FIG. 6.3. Iterations number of ICDD $J_{0, \Gamma}$ with Dirichlet (JGD) and Robin controls with several values of $\beta$ : at left, versus the polynomial degree $p$ for $\mathbb{Q}_{p}$ discretization; at right, versus the overlap thickness $\delta_{12}$, for $\mathbb{Q}_{4}$.

Concerning Robin controls, preliminary results (see [6]) show that they are less efficient than Dirichlet controls, hence the choice of the parameter $\beta$ in (3.4) is not so relevant. Here we will only consider the cost functional $J_{0, \Gamma}$; the other cost functionals display a very similar behavior.

With respect to the polynomial degree $p$ (see Figure 6.3 , left), the larger the $\beta$ the lower the number of iterations. The latter is however higher than that of the ICDD method with Dirichlet controls.

When the overlap thickness $\delta_{12}$ tends to zero and $\beta$ is either null or very small, the number of iterations required by Robin controls is essentially independent of $\delta_{12}$; however for increasing values of $\beta$, the number of iterations increases and shows the same behavior of $J_{0, \Gamma}$ with Dirichlet controls. In any case ICDD with Dirichlet controls turns out to be the most efficient one, as we can see in Figure 6.3, right.

Our conclusion is that the most efficient ICDD method is that obtained by minimizing the cost functional $J_{0, \Gamma}$ (that with interface observation) with Dirichlet controls. From now on we will therefore take into account only such a functional.

Now we consider a decomposition of $\Omega=(0,1)^{2}$ into two subdomains with irregular interfaces as shown in Figure 6.4, and we test the behavior of ICDD with cost functional $J_{0, \Gamma}$, Dirichlet controls, and $\mathbb{P}_{2} \mathrm{FEM}$ discretization, when $\delta_{12}=h$ tends to zero. The mesh is not uniform, nevertheless it is regular and $h$ denotes the maximum diameter of the triangles. 


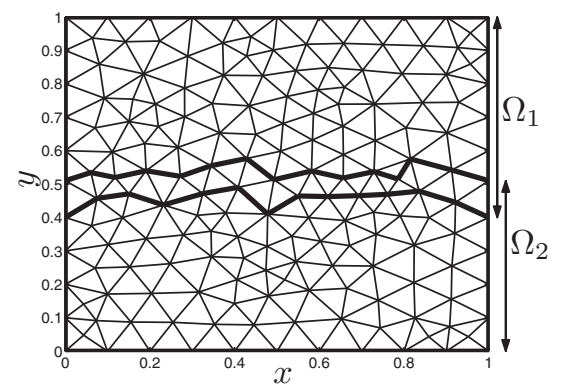

FIG. 6.4. The triangular mesh with irregular interfaces.

TABLE 6.1

Iterations number and errors for the decomposition with irregular interfaces.

$\begin{array}{cccccc}\delta=h & \# \text { iter } & \text { inf } J_{0, \Gamma} & e_{0} & e_{1} & e_{12,0} \\ 1 / 10 & 7 & 6.7834 \mathrm{e}-26 & 6.8367 \mathrm{e}-06 & 5.4954 \mathrm{e}-04 & 6.6013 \mathrm{e}-14 \\ 1 / 20 & 9 & 1.7068 \mathrm{e}-23 & 8.4739 \mathrm{e}-07 & 1.3650 \mathrm{e}-04 & 8.1152 \mathrm{e}-13 \\ 1 / 40 & 13 & 1.6443 \mathrm{e}-24 & 1.0568 \mathrm{e}-07 & 3.3985 \mathrm{e}-05 & 1.7596 \mathrm{e}-13 \\ 1 / 80 & 18 & 1.0253 \mathrm{e}-24 & 1.3228 \mathrm{e}-08 & 8.4858 \mathrm{e}-06 & 8.2360 \mathrm{e}-14\end{array}$

The coefficients of the differential operator are set as follows: $K=1, \mathbf{b}=\mathbf{0}, \gamma=0$; the functions $f$ and $\phi_{D}$ are $f(x, y)=\sin (\pi x) \sin (\pi y)$ and $\phi_{D}=\sin (\pi x) \sin (\pi y) /\left(2 \pi^{2}\right)$.

In Table 6.1 we report the number of ICDD iterations required to satisfy the stopping test on the residual with tolerance $\epsilon=10^{-9}$; the infimum of the cost functional attained at convergence, the errors $e_{0}=\left(\sum_{i=1}^{2}\left\|u-u_{i, h}\right\|_{L^{2}\left(\Omega_{i}\right)}^{2}\right)^{1 / 2}$ and $e_{1}=\left(\sum_{i=1}^{2}\left\|u-u_{i, h}\right\|_{H^{1}\left(\Omega_{i}\right.}^{2}\right)^{1 / 2}$ between the local discrete solutions $u_{i, h}$ and the exact one; the norm of the jump between the local solutions $e_{12,0}=\left\|u_{1, h}-u_{2, h}\right\|_{L^{2}\left(\Omega_{12}\right)}$.

We notice that the number of iterations behaves like \#iter $=C \delta^{-1 / 2}$, as in the case of regular interfaces. The errors with respect to the exact solution decay following theoretical estimates for $\mathbb{P}_{2}$ finite elements, while the error $e_{12,0}$ and the infimum of the cost functional stay within the bounds of rounding errors.

Now we present three test cases, the first one for an operator with discontinuous coefficients; the second one with regular coefficients and a known exact solution for which we test the accuracy in nonconforming discretizations; the third one with dominated convection.

6.1. Test 1. Let us consider the self-adjoint problem

$$
\begin{cases}-\operatorname{div}(K \nabla u)+u=1 & \text { in } \Omega=(0,1)^{2} \\ u=0 & \text { on } \partial \Omega\end{cases}
$$

We investigate the robustness of ICDD with respect to jump discontinuities of the elliptic coefficient $K$. We consider two classical tests, that are usually called central jump and random mix. The computational domain is decomposed into $4 \times 4$ equal subdomains with overlaps of thickness $\delta=0.01$ (equal to $1 \%$ of the side of $\Omega$ ), each subdomain is discretized in $3 \times 3$ square spectral elements, and in each element the spectral polynomial degree is $N=12$ with respect to every spatial variable.

In the central jump test the function $K$ is

$$
K= \begin{cases}\alpha & \text { in } \Omega_{c}=[0.25,0.75]^{2} \\ 1 & \text { in } \Omega \backslash \Omega_{c}\end{cases}
$$




\begin{tabular}{|l|l|l|l|}
\hline $10^{-3}$ & $10^{2}$ & $10^{-6}$ & $10^{2}$ \\
\hline $10^{4}$ & $10^{-1}$ & 1 & $10^{-2}$ \\
\hline $10^{-2}$ & $10^{-5}$ & $10^{2}$ & $10^{-4}$ \\
\hline 1 & $10^{6}$ & $10^{-3}$ & 10 \\
\hline
\end{tabular}

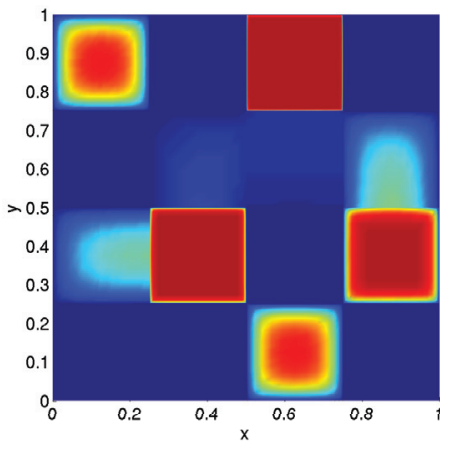

FIG. 6.5. Test case 1, random mix: at left, values of the coefficient $K$ in $\Omega$; at right, the numerical solution.

TABLE 6.2

Test case 1. Iteration counts and infimum of the cost functional for the central jump and random mix configurations.

$\begin{array}{ccc}\alpha & \# \text { it } & \text { inf } J_{0, \Gamma} \\ 10^{-6} & 16 & 2 \cdot 10^{-22} \\ 10^{-4} & 17 & 1 \cdot 10^{-24} \\ 10^{-2} & 20 & 1 \cdot 10^{-25} \\ 1 & 28 & 6 \cdot 10^{-26}\end{array}$

$\begin{array}{ccc}\alpha & \# \text { it } & \text { inf } J_{0, \Gamma} \\ 10^{2} & 20 & 1 \cdot 10^{-25} \\ 10^{4} & 24 & 5 \cdot 10^{-23} \\ 10^{6} & 23 & 1 \cdot 10^{-22} \\ \text { random } & 9 & 9 \cdot 10^{-21}\end{array}$

with $\alpha$ varying from $10^{-6}$ up to $10^{6}$. In the random mix case, $K$ is defined as in the left picture of Figure 6.5.

In Table 6.2 we report the iteration counts and the infimum of the cost functional $J_{0, \Gamma}$ obtained at convergence, for different values of the parameter $\alpha$. The iteration counts refer to Bi-CGSTAB, called here to solve the Schur complement system associated with the discretization of (4.14). The stopping test is satisfied when the norm of the residual is reduced of 12 orders of magnitude.

The results show that the convergence rate of ICDD to the solution of the minimum problem (3.7) is independent of the jumps of the coefficients.

The size of the overlap is responsible in general for the convergence rate of the Bi-CGSTAB iterations and, in the case of a discontinuous coefficient, also for the accuracy of the approximation. More precisely, if the jump of the coefficient is very large, the high variation of the solution is correctly captured without oscillations only if the discretization is fine enough in a small region around the jump. We can achieve good results, e.g., by using a very small overlap, then discretizing the overlap with one spectral element (along the direction across the jump) and by using a moderately large value of the polynomial degree $N$. Otherwise we can use a generous overlap in spite of adopting higher polynomial degree $N$. On one hand, the smaller the overlap thickness, the slower the convergence rate to the minimum point. On the other hand, the larger the polynomial degree $N$, the more expensive the solution of the boundary value problems inside each subdomain. Therefore, a careful tuning of the discretization parameters is in order to minimize the computational costs without compromising accuracy or stability.

6.2. Test 2. Let us consider now problem $(2.2)$ in $\Omega=(0,3)^{2}$, with Dirichlet conditions $u=\phi_{D}$ on the boundary. We take $K=1, \mathbf{b}=(y-1, x), b_{0}=1$, while the functions $f$ and $\phi_{D}$ are chosen so that the exact solution is $u(x, y)=$ 

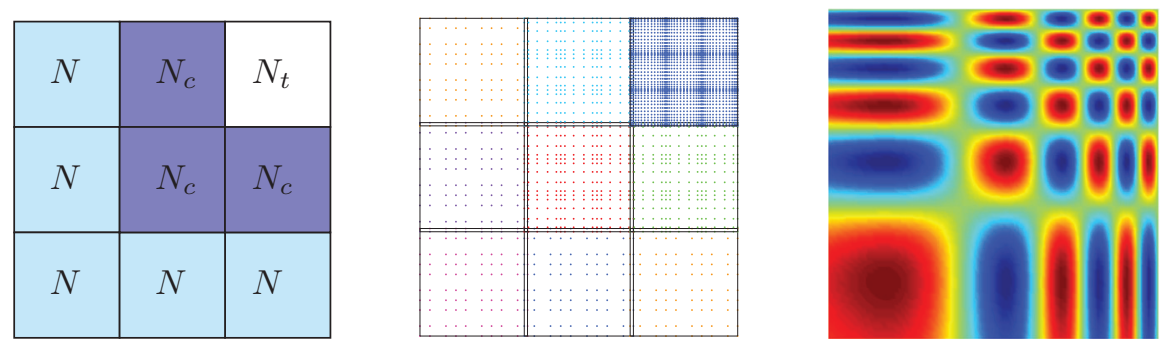

FIG. 6.6. Test case 2. At left, subdomains with the same color share the same discretization (the same number of spectral elements and the same polynomial degree). At center, is a possible nonconforming mesh, the overlaps are marked with black lines. At right, is the numerical solution with $N=12, N_{c}=14$, and $N_{t}=16$.

TABLE 6.3

Test case 2. Iteration counts and absolute error with respect to the exact solution in $H^{1}$ norm. $N$ denotes the polynomial degree in the subdomains of the left-bottom L-shaped band. At left, the grids do not match on the overlaps for any $N$, also the number of elements differ band by band. At right, the number of spectral elements is the same in each subdomain, but the grids match only when $N=16$.

$\begin{array}{cccccccccc}N & N_{c} & N_{t} & \# \text { it } & H^{1} \text {-error } & N & N_{c} & N_{t} & \# \text { it } & H^{1} \text {-error } \\ 4 & 6 & 8 & 29 & 1.44 \cdot 10^{-1} & 6 & 8 & 16 & 27 & 2.50 \cdot 10^{-3} \\ 6 & 8 & 10 & 26 & 8.89 \cdot 10^{-3} & 8 & 10 & 16 & 26 & 4.49 \cdot 10^{-5} \\ 8 & 10 & 12 & 27 & 1.46 \cdot 10^{-4} & 10 & 12 & 16 & 27 & 3.30 \cdot 10^{-7} \\ 10 & 12 & 14 & 27 & 1.25 \cdot 10^{-5} & 12 & 14 & 16 & 26 & 1.63 \cdot 10^{-8} \\ 12 & 14 & 16 & 26 & 3.76 \cdot 10^{-7} & 14 & 16 & 16 & 27 & 1.61 \cdot 10^{-10} \\ 14 & 16 & 20 & 27 & 1.94 \cdot 10^{-9} & 16 & 16 & 16 & 26 & 7.79 \cdot 10^{-12}\end{array}$

$\sin \left(6 \pi e^{x-3}\right) \sin \left(6 \pi e^{y-3}\right)$. This function shows a discrete number of bumps that are mainly located in the square $(2,3) \times(2,3)$ (see Figure 6.6 , right). Thus, it is natural to enrich the discretization in the computational domain when moving from left to right and from bottom to top. To realize this, we decompose $\Omega$ in $3 \times 3$ square subdomains $\Omega_{k}$ with a small overlap of size $\delta$ (that will be specified later). In its turn, each subdomain $\Omega_{k}$ is discretized in $n e_{k} \times n e_{k}$ spectral elements with polynomial degree $N_{k}$ with respect to both $x$ and $y$. The number of spectral elements and the polynomial degree can differ from one subdomain to another, providing nonmatching grids on the overlaps. We analyze the convergence of the numerical solution towards the exact one in $H^{1}$-norm for various nonconforming discretizations.

We fix the overlap thickness equal to $\delta=0.03$. In each subdomain of the leftbottom L-shaped band (the light-blue region of the picture at left of Figure 6.6) we consider $3 \times 3$ spectral elements with polynomial degree $N$, in each subdomain of the central L-shaped band (blue region) we take $4 \times 4$ spectral elements with polynomial degree $N_{c}=N+2$, and, finally, in the top-right subdomain we have $5 \times 5$ spectral elements with polynomial degree $N_{t}=N+4$ (see Figure 6.6, at center).

As we can read in Table 6.3 (at left), the error decays with exponential accuracy when the polynomial degree $N$ (and consequently also $N_{c}$ and $N_{t}$ ) grows up, as it typically happens for conforming spectral elements approximations. We remark the fact that an interpolation technique between one spectral element grid to the other has been used to match the two solutions on the interfaces of the overlaps.

In Table 6.3 (at right) we show the $H^{1}$-norm error with respect to the exact solution, for another discretization. The domain $\Omega=(0,3)^{2}$ is split again in $3 \times 3$ 

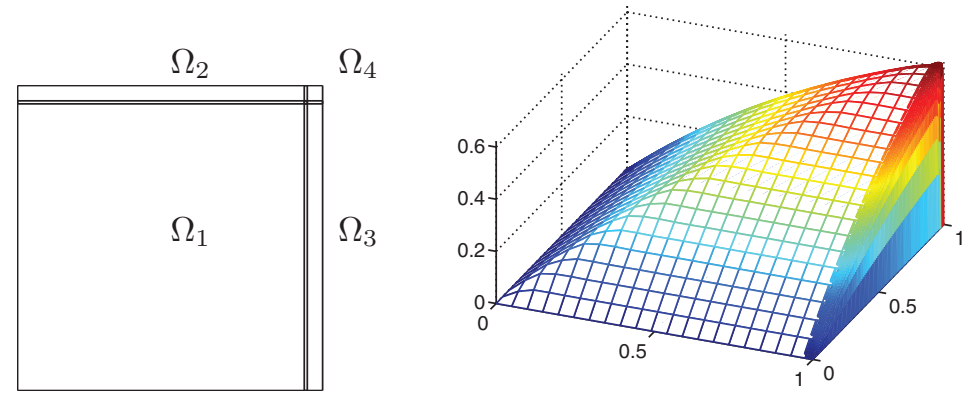

FIG. 6.7. Test case 3: at left, the decomposition of the computational domain; at right, the numerical solution of the heterogeneous coupling with $K=10^{-6}$, obtained by stabilized $\mathbb{Q}_{2}$ finite elements.

square subdomains, but we consider now $4 \times 4$ spectral elements in each subdomain $\Omega_{k}$ (for $k=1, \ldots, 9$ ), while the polynomial degree is equal to $N$ in each subdomain of the left-bottom L-shaped band, equal to $N_{c}=\min \{N+2,16\}$ in each subdomain of the centered L-shaped band, and equal to $N_{t}=16$ in the top-right subdomain. The discretization is conforming only when $N=16$. Also in this case we recover exponential $H^{1}$-convergence of the numerical solution to the exact one.

The number of Bi-CGSTAB iterations required to converge to the minimum point of (3.7) is independent of the polynomial degree $N$. In [6] the convergence rate of ICDD methods will be analyzed with respect to the other discretization parameters, as the number of subdomains $M$, the size of the overlap, and the grid size for finite element discretizations.

6.3. Test 3. Let us consider problem $(2.2)$ in $\Omega=(0,1)^{2}$, where $K$ is a small positive constant, $\mathbf{b}=[1,1]^{t}, b_{0}=1, f=1$, and $\phi_{D}=0$. It is a convection-dominated problem whose solution features boundary layers on the top and on the right sides of the computational domain [18]. We analyze the behavior of the ICDD method (4.14) when the elliptic coefficient $K$ varies from $10^{-6}$ to $10^{-2}$. Moreover, for any value of $K$ considered, we solve the heterogeneous A-AD coupling (5.1)-(5.2) by the ICDD method (5.6) (or equivalently (5.8)) by setting $K=0$ in a subregion of the domain far from the layers. We measure the difference in $L^{2}$-norm between the state solution of the homogeneous ICDD (with elliptic problems in all the subdomains) and that of the heterogeneous ICDD, as well as the efficiency of the ICDD method in terms of iterations count.

We split the computational domain into $2 \times 2$ subdomains whose interfaces are close to the boundary layers (see Figure 6.7, left). We set $\Omega_{1}=\left(0, x_{\Gamma}+\delta / 2\right) \times$ $\left(0, y_{\Gamma}+\delta / 2\right), \Omega_{2}=\left(0, x_{\Gamma}+\delta / 2\right) \times\left(y_{\Gamma}-\delta / 2,1\right), \Omega_{3}=\left(x_{\Gamma}-\delta / 2,1\right) \times\left(0, y_{\Gamma}+\delta / 2\right)$, and $\Omega_{4}=\left(x_{\Gamma}-\delta / 2,1\right) \times\left(y_{\Gamma}-\delta / 2,1\right)$, the thickness of the overlap is $\delta=0.01$ (corresponding to $1 \%$ of the side of the computational domain) for all the cases, while $x_{\Gamma}=y_{\Gamma}$ will be specified later and they will be chosen so that they do not fall in the boundary layer region. In the heterogeneous case, we solve the hyperbolic equation in the subdomain $\Omega_{1}$ and the elliptic equation in $\widetilde{\Omega}_{2}=\cup_{k=2}^{4} \Omega_{k}$.

In each subdomain we discretize the boundary value problems by $\mathbb{Q}_{2}$ finite elements, stabilized with Galerkin Least Squares techniques (see [9]) for the elliptic case. In Table 6.4 we report the number of Bi-CGSTAB iterations required to solve the Schur complement of system (2.2) up to reducing the residual of 12 orders of magnitude for both homogeneous $\left(\# i t_{e}\right)$ and heterogeneous $\left(\# i t_{h}\right)$ couplings. By denoting 
TABLE 6.4

Test case 3. Iterations count and errors for the convection dominated solution.

$\begin{array}{llllll}K & x_{\Gamma} & \# i t_{h} & \# i t_{e} & \left\|u_{e}-u_{h}\right\|_{L^{2}\left(\Omega_{1}\right)} & \left\|u_{e}-u_{h}\right\|_{L^{2}\left(\widetilde{\Omega}_{2}\right)} \\ 10^{-2} & 0.9 & 6 & 9 & 9.48 \cdot 10^{-5} & 1.61 \cdot 10^{-5} \\ 10^{-3} & 0.95 & 5 & 11 & 2.38 \cdot 10^{-6} & 2.41 \cdot 10^{-7} \\ 10^{-4} & 0.95 & 7 & 13 & 1.83 \cdot 10^{-7} & 1.10 \cdot 10^{-8} \\ 10^{-5} & 0.95 & 7 & 13 & 1.49 \cdot 10^{-7} & 7.10 \cdot 10^{-9} \\ 10^{-6} & 0.98 & 6 & 13 & 1.48 \cdot 10^{-7} & 6.90 \cdot 10^{-9}\end{array}$

with $u_{e}$ and $u_{h}$ the state solutions of the homogeneous and heterogeneous couplings, respectively, we report the errors $\left\|u_{e}-u_{h}\right\|_{L^{2}\left(\Omega_{1}\right)}$ and $\left\|u_{e}-u_{h}\right\|_{L^{2}\left(\widetilde{\Omega}_{2}\right)}$. Numerical results show that, for any considered value of $K$, to solve the heterogeneous problem instead of the homogeneous one is advantageous and the differences between the heterogeneous and homogeneous solutions vanish when $K$ tends to zero. The mesh in $\Omega_{1}$ is fixed in $10 \times 10$ elements, while in the other subdomains we consider different meshes versus the values of $K$. More precisely, we fix $50 \times n_{K}$ elements in $\Omega_{2}, n_{K} \times 50$ in $\Omega_{3}$, and $n_{K} \times n_{K}$ in $\Omega_{4}$, with $n_{K}=5$ when $K=10^{-2}, 10^{-3}, 10^{-4}$ and $n_{K}=10$ when $K=10^{-5}, 10^{-6}$. In all cases the meshes are nonmatching on the overlaps.

7. Conclusion. In this paper we have introduced and analyzed the ICDD method for the mathematical formulation and the numerical solution of elliptic partial differential equations. The idea consists of reformulating the original boundary value problem on a decomposition of the domain $\Omega$ with overlapping subdomains $\Omega_{k}$; introducing as control variables the unknown traces (of Dirichlet, Neumann, or Robin type) of the original solution at the internal boundaries of the subdomains $\Omega_{k}$; introducing a cost functional expressing the minimization of a suitable interface jump; deriving the associated optimality system. The latter is numerically solved. The results obtained on three different PDEs highlight the excellent properties of efficiency of the ICDD method, especially its robustness with respect to the variation of the physical coefficients. Of remarkable interest is the accuracy and computational efficiency of the ICDD method to deal with heterogeneous PDEs (A-AD problems considered here, Stokes-Darcy problems that will be dealt with in [7]).

\section{REFERENCES}

[1] V.I. Agoshkov, P. Gervasio, And A. Quarteroni, Optimal control in heterogeneous domain decomposition methods for advection-diffusion equations, Mediterr. J. Math., 3 (2006), pp. 147-176.

[2] P.J. Blanco, P. Gervasio, and A. Quarteroni, Extended variational formulation for heterogeneous partial differential equations, Comput. Methods Appl. Math., 11 (2011), pp. 141172.

[3] C. Canuto, M. Y. Hussaini, A. Quarteroni, and T.A. Zang, Spectral Methods. Evolution to Complex Geometries and Applications to Fluid Dynamics, Springer, Heidelberg, 2007.

[4] M. Discacciati, P. Gervasio, and A. Quarteroni, Heterogeneous mathematical models in fluid dynamics and associated solution algorithms, in Multiscale and Adaptivity, Lecture Notes in Math. 2040, Springer, Heidelberg, 2011, pp. 57-123.

[5] M. Discacciati, P. Gervasio, and A. Quarteroni, Convergence Analysis of Interface Control Domain Decomposition (ICDD) Methods for Elliptic Problems, Technical report, MOX Politecnico di Milano, 2013, in preparation.

[6] M. Discacciati, P. Gervasio, and A. Quarteroni, Interface Control Domain Decomposition (ICDD) Methods for Coupled Diffusion and Advection-Diffusion Problems, Int. J. Numer. Methods Fluids, submitted. 
[7] M. Discacciati, P. Gervasio, and A. Quarteroni, Interface Control Domain Decomposition Methods for the Stokes-Darcy Coupling, Technical report, MOX - Politecnico di Milano, 2013, in preparation.

[8] M. Discacciati And A. Quarteroni, Navier-Stokes/Darcy coupling: Modeling, analysis, and numerical approximation, Rev. Mat. Complut., 22 (2009), pp. 315-426.

[9] L.P. Franca, S.L. Frey, and T.J.R. Hughes, Stabilized finite element methods: I. Application to the advective-diffusive model, Comput. Methods Appl. Mech. Engrg., 95 (1992), pp. 253276.

[10] F. Gastaldi And A. Quarteroni, On the coupling of hyperbolic and parabolic systems: Analytical and numerical approach., Appl. Numer. Math., 6 (1989), pp. 3-31.

[11] F. Gastaldi, A. Quarteroni, and G. Sacchi Landriani, On the coupling of two dimensional hyperbolic and elliptic equations: Analytical and numerical approach, in 3rd International Symposium on Domain Decomposition Methods for Partial Differential Equations, J.Périeaux T.F.Chan, R.Glowinski and O.B. Widlund, eds., SIAM, Philadelphia, 1990, pp. $22-63$.

[12] P. Gervasio, J.-L. Lions, and A. Quarteroni, Heterogeneous coupling by virtual control methods, Numer. Math., 90 (2001), pp. 241-264.

[13] R. Glowinski, Q.V. Dinh, and J. Periaux, Domain decomposition methods for nonlinear problems in fluid dynamics, Comput. Methods Appl. Mech. Engrg., 40 (1983), pp. 27-109.

[14] P. Grisvard, Elliptic Problems in Nonsmooth Domains, Pitman, Boston, MA, 1985.

[15] J.-L. Lions, Optimal Control of Systems Governed by Partial Differential Equations, SpringerVerlag, New York, 1971.

[16] J.-L. Lions And O. Pironneau, Algorithmes parallèles pour la solution de problèmes aux limites, C. R. Acad. Sci. Paris Sér. I Math., 327 (1998), pp. 947-952.

[17] E. Miglio, A. Quarteroni, And F. Saleri, Coupling of free surface and groundwater flows, Comput. \& Fluids, 32 (2003), pp. 73-83.

[18] A. Quarteroni, Numerical Models of Differential Problems, Series MS\&A 2, Springer, Milan, 2009.

[19] A. Quarteroni and A. Valli, Domain Decomposition Methods for Partial Differential Equations, Clarendon Press, Oxford, 1999.

[20] M. Signorini, Interface Control Domain Decomposition Problems, Master's thesis, Mathematical Engineering, Politecnico di Milano, Milan, 2012.

[21] A.E. TAYLOR, Introduction to Functional Analysis, John Wiley, New York, 1958.

[22] A. Toselli And O. Widlund, Domain decomposition methods-algorithms and theory, Springer Ser. Comput. Math. 34, Springer-Verlag, Berlin, 2005.

[23] H.A. van Der Vorst, Iterative Krylov methods for large linear systems, Cambr. Monogr. Appl. Comput. Math. 13, Cambridge University Press, Cambridge, 2003. 